\title{
MODELLING AND CONTROL OF AN OMNIDIRECTIONAL MOBILE MANIPULATOR
}

\author{
Salima DJEBRANI ${ }^{* * *}$, AbderRaouf BENALI ${ }^{* *}$, Foudil ABDESSEMED* \\ * Department of Electronics \\ University of Batna, Rue Chahid Boukhlouf, Batna 05000, Algeria \\ e-mail: nawa74dz@yahoo.fr \\ **ENSI de Bourges \\ PRISME, 10 Boulevard Lahitolle, 18020 Bourges cedex, France
}

\begin{abstract}
A new approach to control an omnidirectional mobile manipulator is developed. The robot is considered to be an individual agent aimed at performing robotic tasks described in terms of a displacement and a force interaction with the environment. A reactive architecture and impedance control are used to ensure reliable task execution in response to environment stimuli. The mechanical structure of our holonomic mobile manipulator is built of two joint manipulators mounted on a holonomic vehicle. The vehicle is equipped with three driven axles with two spherical orthogonal wheels. Taking into account the dynamical interaction between the base and the manipulator, one can define the dynamics of the mobile manipulator and design a nonlinear controller using the input-state linearization method. The control structure of the robot is built in order to demonstrate the main capabilities regarding navigation and obstacle avoidance. Several simulations were conducted to prove the effectiveness of this approach.
\end{abstract}

Keywords: holonome mobile manipulators, input state linearization, virtual impedance control, fuzzy logic.

\section{Introduction}

To date, there have been a lot of research efforts regarding developing mobile mechanisms, which can be categorized into three types: vehicles equipped with wheels similar to general (automobile-type) vehicles, with two parallel wheels and one caster wheel, and the ones with omnidirectional wheels (Campion et al., 1996). Automobiletype vehicles cannot perform rotation or side-translational motion in a narrow space; vehicles with two parallel wheels and one caster mechanism cannot perform sidetranslational motion either.

The omni-directional mobile robot is a kind of holonomic robot. Compared with the more common car like (nonholonomical) mobile robot, the omni-directional one has the ability to move simultaneously and independently in translation and rotation (Pin and Killough, 1994). Most of the work on motion control of omnidirectional mobile robots is based mainly on the kinematic model. This is equivalent to assuming that robots are massless bodies and therefore can ideally respond to the input motion control commands, which indeed does not reflect the real situation especially for heavy and fast moving robots. For this reason, efforts have been made to develop precise dynamic models to improve robot performance (Williams et al., 2002; Watanabe et al., 1998).

One of the main problems in robotic research is to provide efficient control algorithms in order to allow robots to execute desired tasks. These tasks are various and require great mobility and dexterity of the robotic system. A mobile manipulator is generally one of the structures aimed at these applications. It is basically a robotic arm mounted on a moving base and can be used to perform a variety of tasks that are mostly related to material handling application. The capability of mobile manipulation is important for some autonomous mobile robot applications, which need to interact with the environment dynamically. These applications require both a large workspace and a dexterous manipulation capability. Application examples in servicing robotics include the ARMAR robot (Albers et al., 2006), ROBONAUT (Ambrose et al., 2004), HERMES (Bischoff and Graefe, 2004), HADALY-2 (Hashimoto, 2002), the mobile robot HELPER (Kosuge et al., 2000) or SAIKA (Konno et al., 1997), etc. The mobility of the mobile 
platform substantially increases the performance capabilities of the system. For example, the platform increases the size of the manipulator workspace and enables the manipulator to position the end-effector for executing the task efficiently. Because of the kinematic redundancy and mobility, the functionality of the mobile manipulator is increased to a greater extent (Abdessemed et al., 2006; Bayle, 2001; Yamamoto, 1994).

Mobile manipulators are often equipped with wheels. The arrangement of the wheels and their actuation device determine the holonomic or nonholonomic nature of its locomotion system (Campion et al., 1996), whereas some wheeled mobile manipulators built of an omnidirectional platform are holonomic (Khatib et al., 1996). While the mobile manipulator provides numerous advantages compared with the fixed base manipulator, path planning and control of such a system are challenging problems. First, there is the problem of redundancy and coordination. However, coordination can be performed by using kinematic or dynamic models. The dynamic model of a mobile manipulator is complex and there is a high degree of coupling between the platform and the arm. The dynamics of the mobile platform and the manipulator arm are quite different. The base is heavy and has slow dynamics. The arm is relatively light and has fast dynamics. Such a system can provide precise motion of the arm for local operation and retain the versatility and workspace of the mobile platform. In this paper, our mobile manipulator is holonomic. It is built of a two degree manipulator mounted on a holonomic structure equipped with three motorized axles with two spherical orthogonal wheels (Mourioux et al., 2006; Poisson et al., 2001). In this work we aim to control the whole dynamics of such a structure in terms of state space feedback.

Feedback linearization methods can be viewed as ways of algebraically transforming a fully or partially nonlinear dynamic system into a simple linear one. In the standard approach to exact input-state linearization, one uses coordinate transformation and static state feedback such that the closed-loop system, in the defined region, takes a linear canonical form. After the system's linearized form is obtained, the linear control design scheme is employed to achieve stabilization or tracking of a desired trajectory (Isidori, 1995; Slotine and Li, 1991). This scheme is used in local coordinate linearization with an outer loop aimed at avoiding collisions with obstacles. A sensorial system should detect an obstacle and measure its distance to calculate a control action to change the mobile robot trajectory, thus avoiding any obstacles. Most works in this area consider motion control of a mobile robot while avoiding obstacles (Carelli et al., 1999; Borenstein and Koren, 1991; Khatib, 1986; Hogan, 1985). In order to unify our approach, we propose to use the impedance control during obstacle avoidance. The control architecture combines two loops: a motion control loop and a sec- ond external impedance control loop. The latter provides a modification of the trajectory of the mobile platform when an obstacle appears on the trajectory of the mobile manipulator. The main contributions of this paper include: the proposal of a new architecture of the robot, designing a control structure for obstacle avoidance, and determining the corresponding impedance parameters using fuzzy logic.

The paper is organized in as follows. Section 2 is aimed to present the robot architecture. Sections 3 and 4 present the kinematic and dynamic models of the robot manipulator and the omnidirectional mobile platform. The model of the omnidirectional mobile manipulator is developed in Section 5. In Section 6, a local coordinates linearization controller for the mobile manipulator is developed. The concept of virtual impedance control is presented in Section 7. In Section 8, simulation tests are presented to show the performance of the control algorithms. Section 9 concludes the paper.

\section{Robot structure and architecture}

We consider a mobile manipulator depicted in Fig. 8. It consists of an omnidirectional mobile platform (Figs. 3 and 4) and a two-link manipulator (Fig. 2). The platform moves by driving the three wheels. This mechanical structure is monitored by using a design of a distributed multi-tasking environment. This environment offers multi-thread programming capabilities and interprocess communication message protocols. The design of the mobile manipulator structure must integrate multiple capabilities such as navigation, manipulation and interaction with the environment. The mobile manipulator has to avoid static/dynamic obstacles as well as collaborate with other robots or humans. Such behaviors have to be organized in order to achieve a variety of tasks. The multi-agent structure offers the appropriate approach. The robot is autonomous and can communicate with the others in order to synchronize its response according to the required task. In our approach the mobile manipulator represents an agent (Djebrani et al., 2010a; Djebrani and Abdessemed, 2009). Its structure is organized as in Fig. 1.

The robot structure is composed mainly of the following modules:

- Control mode manager. This module ensures the management of the control law selection. Thus, we defined the generic organization of a multiple layer structure. Each layer provides a specific controller. By means of this module the robot can select different strategies (free navigation, obstacle avoidance, etc.) or alternate the roles when cooperating with other robots.

- Communication module. The objective of this 


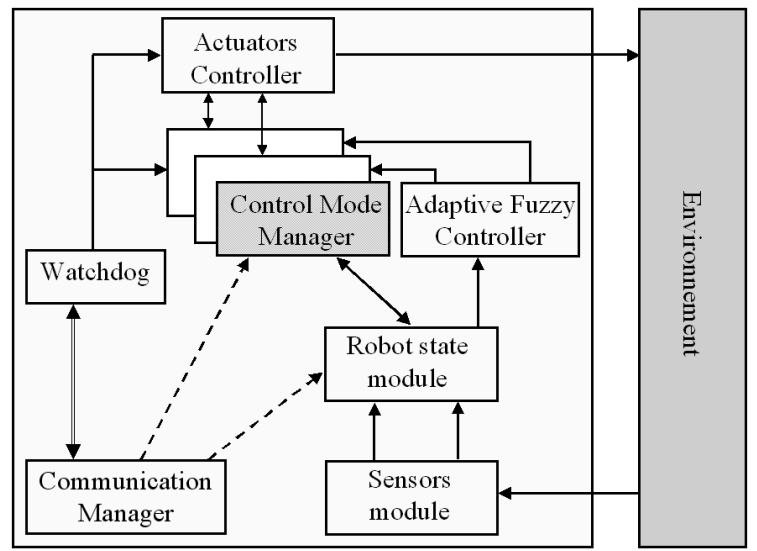

Fig. 1. Robot architecture.

module is twofold: it permits to communicate with other agents and gives the human operator all the information needed to monitor the state of the robot.

- Sensors module. This module senses the robot and the environment. It collects all data exchanges with the robot state module. In our application we considered ultrasonic sensors implemented as shown in Fig. 6. Ultrasonic sensors are arranged in front of the robot and two adjacent sensors are at a distance of 45 degrees from each other. The maximum range of each sensor slightly exceeds $3 \mathrm{~m}$. At a fixed sampling time the module measures and transmits the necessary information about position, velocity, etc. to the robot state module.

- Adaptive fuzzy controller. This module is aimed to tune the parameters of the impedance used to control the robot displacement. This module is summarized in Section 7.

- Watchdog module. To ensure the safety of the robot, this module detects all the communication errors during the robotics tasks.

- Robot state module. This module includes all information from the sensors module and the communication manager module. It manages data collection and its storage in the controller memory.

- Actuators controller module. This module takes care of sending to the actuators the current desired velocities. It provides low-level control of the robot depending on the chosen mode designated by the control mode manager module.

All the described modules are used to control the robot's behavior. In the next section we shall present robot modelling in order to develop the control law implementation used in the control mode manager.

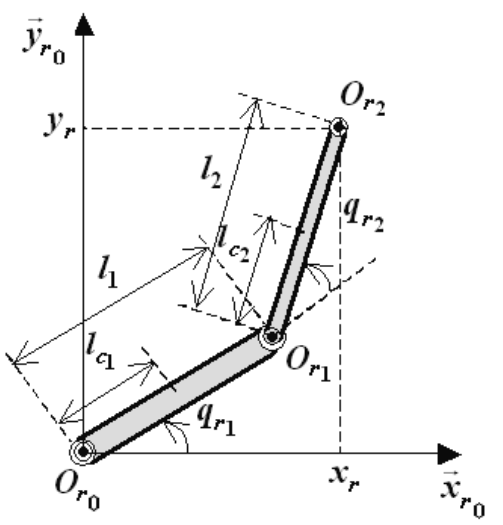

Fig. 2. Two-link revolute arm.

\section{Robot manipulator modelling}

A robotic manipulator with $n_{r}$ degrees of freedom joints can be modeled as a set of rigid bodies connected in a series with one end fixed to the ground and the other end free (Sciavicco and Siciliano, 2000; Spong et al., 1989; Khalil and Kleinfinger, 1986). The bodies are actuated with revolute or prismatic joints. A two-link planar $R R$ robot arm is shown in Fig. 2. The robot arm is restricted to move in the plane. The robotic arm configuration is given by the rotational angles $q_{r_{1}}$ and $q_{r_{2}}$. Let us define the robot joint vector $q_{r}=\left[q_{r_{1}}, q_{r_{2}}\right]^{T}$ of dimension $n_{r}=2$, and let us define the end-effector location by the planar position of $O_{r_{2}}$ in $R_{r_{0}}: \xi_{r}=\left[x_{r}, y_{r}\right]^{T}$ of dimension $m_{r}=2$. The robotic arm KM (Kinematic Model) is

$$
\left\{\begin{array}{l}
x_{r}=l_{1} \cos \left(q_{r_{1}}\right)+l_{2} \cos \left(q_{r_{1}}+q_{r_{2}}\right), \\
y_{r}=l_{1} \sin \left(q_{r_{1}}\right)+l_{2} \sin \left(q_{r_{1}}+q_{r_{2}}\right) .
\end{array}\right.
$$

The dynamic equation of the manipulator using the Lagrange formalism is given by

$$
M_{r}\left(q_{r}\right) \dot{\omega}_{r}+C_{r_{1}}\left(q_{r}, \omega_{r}\right) \omega_{r}=\tau_{r},
$$

where the $n_{r} \times n_{r}$ "inertia matrix" $M_{r}\left(q_{r}\right)$ is symmetric and positive definite for each $q_{r} \in \mathbb{R}^{n_{r}}, C_{r_{1}}\left(q_{r}, \omega_{r}\right)$ is the Coriolis/centrifugal matrix. The inputs $\tau_{r_{i}}$ to the system are the torques applied to each arm. The dynamic equation of the planar robot manipulator is derived by using the Euler-Lagrange method giving

$$
\begin{aligned}
& {\left[\begin{array}{cc}
M_{r}^{(11)} & M_{r}^{(12)} \\
M_{r}^{(21)} & M_{r}^{(22)}
\end{array}\right]\left[\begin{array}{c}
\dot{\omega}_{r_{1}} \\
\dot{\omega}_{r_{2}}
\end{array}\right]} \\
& +\left[\begin{array}{cc}
h \omega_{r_{2}} & h\left(\omega_{r_{1}}+\omega_{r_{2}}\right) \\
-h \omega_{r_{1}} & 0
\end{array}\right]\left[\begin{array}{l}
\omega_{r_{1}} \\
\omega_{r_{2}}
\end{array}\right]=\left[\begin{array}{c}
\tau_{r_{1}} \\
\tau_{r_{2}}
\end{array}\right],
\end{aligned}
$$



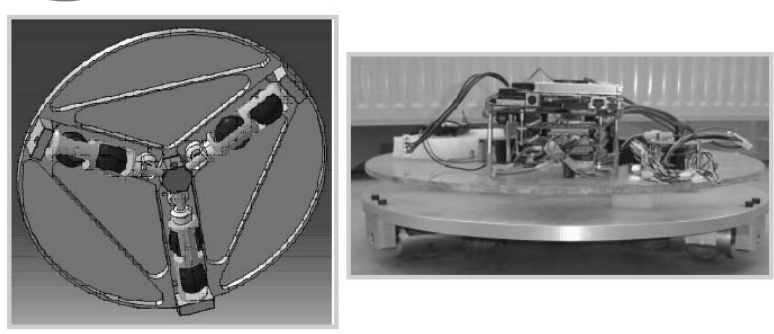

Fig. 3. ROMNI: omnidirectional robot.

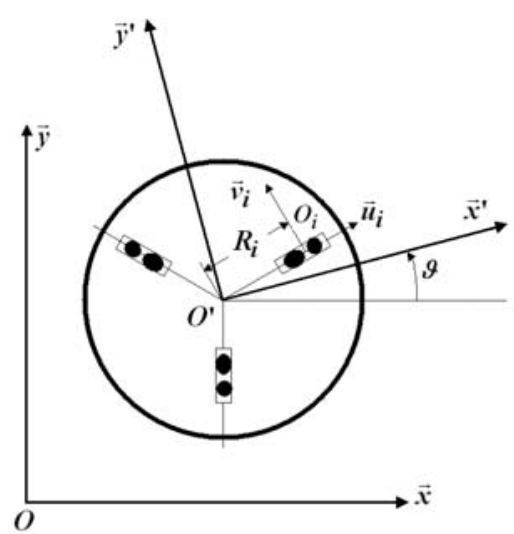

Fig. 4. Absolute frame, robot frame and axle frame.

$$
\begin{aligned}
\omega_{r}= & {\left[\omega_{r_{1}}, \omega_{r_{2}}\right]^{T}=\dot{q}_{r}=\left[\dot{q}_{r_{1}}, \dot{q}_{r_{2}}\right]^{T}, } \\
\tau_{r}= & {\left[\tau_{r_{1}}, \tau_{r_{2}}\right]^{T}, } \\
M_{r}^{(11)}= & m_{1} l_{c_{1}}^{2}+m_{2}\left(l_{1}^{2}+l_{c_{2}}^{2}+2 l_{1} l_{c_{2}} \cos \left(q_{r_{2}}\right)\right) \\
& +I_{1}+I_{2}, \\
M_{r}^{(12)}= & M_{r}^{(21)}=m_{2}\left(l_{c_{2}}^{2}+l_{1} l_{c_{2}} \cos \left(q_{r_{2}}\right)\right)+I_{2}, \\
M_{r}^{(22)}= & m_{2} l_{c_{2}}^{2}+I_{2}, \\
h= & -m_{2} l_{1} l_{c_{2}} \sin \left(q_{r_{2}}\right),
\end{aligned}
$$

where $q_{r_{i}}$ denotes the joint angle, $m_{i}$ denotes the mass of Link $i, l_{i}$ denotes the length of Link $i, l_{c_{i}}$ denotes the distance from the previous joint to the center of mass of Link $i$ and $I_{i}$ denotes the moment of inertia of Link $i$.

\section{Omnidirectional mobile robot modelling}

This section presents kinematic and dynamic modelling of the ROMNI omnidirectional robot. This robot was developed at the Bourges PRISME laboratory (Mourioux et al., 2006; Poisson et al., 2001). As a first step to develop a robot controller, the equations of robot motion have to be derived.

4.1. Kinematic modelling. Figure 3 shows the bottom view of the ROMNI robot. This robot has a mechanical

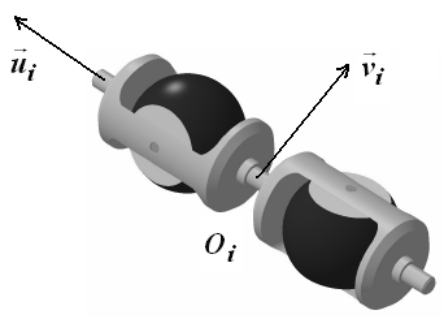

Fig. 5. Axle with longitudinal orthogonal wheels.

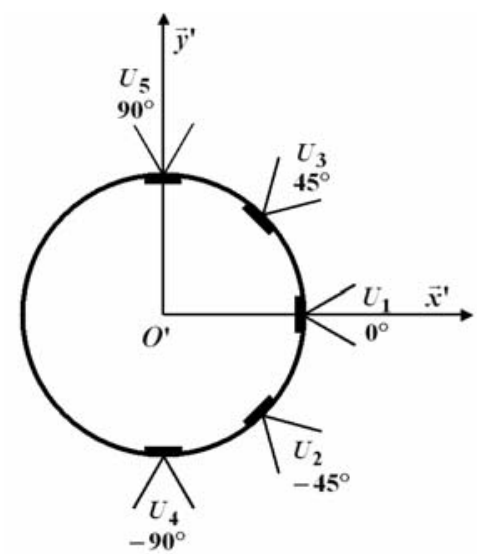

Fig. 6. Robot and its ultrasonic perception system.

structure that enables it to change its displacement direction at any moment, without reconfiguring its rolling parts (Mourioux et al., 2006; Poisson et al., 2001). The axle is composed of two orthogonal wheels with a phase of $\pi / 2$ (Figs. 4 and 5). The reader can refer to the works of Mourioux et al. (2006) and Poisson et al. (2001) for more details on an exact description of this structure. The posture is defined in Fig. 4, where $(O, \vec{x}, \vec{y})$ is the world coordinate system, $O$ is the reference point, $\left(O^{\prime}, \vec{x}^{\prime}, \vec{y}^{\prime}\right)$ is the robot coordinate system, and the point $O^{\prime}$ is the center of the robot.

In order to determine the kinematic model of the platform, one can calculate the velocity $\vec{V}_{O_{i}}$ of the contact point of the wheel with the floor $\left(O_{i}\right.$ in Figs. 4 and 5):

$$
\begin{aligned}
\vec{V}_{O_{i}} & =\vec{V}_{O^{\prime}}+\vec{\Omega} \wedge \overrightarrow{O^{\prime} O_{i}} \\
& =\dot{x} \vec{x}+\dot{y} \vec{y}+\dot{\vartheta} R_{i} \vec{v}_{i}
\end{aligned}
$$

If we assume that the wheels do not slip, the relative velocity of the wheel-to-floor contact point is zero. Then for each contact point $O_{1}, O_{2}$ and $O_{3}$, we can write

$$
\vec{V}_{O_{i}} \vec{v}_{i}=-r \dot{\varphi}_{i}, \quad \forall i \in\{1,2,3\} .
$$

We use the following notation: $\vec{u}_{i}$ and $\vec{v}_{i}$ are the vectors of longitudinal direction of the $i$-th axle and its perpendicular, respectively; $O_{i}$ is the contact point of the 
wheel with the floor; $\vec{V}_{O_{i}}$ is the velocity of the point $O_{i}$; $\vec{V}_{O^{\prime}}$ is the velocity of the point $O^{\prime} ; \vec{\Omega}$ stands for the angular velocity of the $O^{\prime}$ point; $\dot{\varphi}_{i}$ is the angular velocity of the $i$ axle; $R_{i}$ is the variable distance $O^{\prime} O_{i} ; \vartheta$ is the angle between $\vec{x}$ and $\vec{x}^{\prime}$ (it characterizes the absolute orientation of the robot); $r$ is the radius of the sphere; $\alpha_{i}$ is the angle between $\vec{x}^{\prime}$ and $\vec{u}_{i}, \alpha_{1}=0, \alpha_{2}=2 \pi / 3, \alpha_{3}=4 \pi / 3$; $\dot{x}, \dot{y}, \dot{\vartheta}$ are the kinematic parameters of the motion at the point $O^{\prime}$ of the platform expressed in the absolute reference frame linked to the environment $(O, \vec{x}, \vec{y})$.

First, consider the following three-dimensional vector describing the robot:

$$
\xi_{p}=[x, y, \vartheta]^{T},
$$

where $x$ and $y$ are the coordinates related to the reference $O^{\prime}$ in the world frame. Second, write

$$
\omega_{v}=\left[\omega_{v_{1}}, \omega_{v_{2}}, \omega_{v_{3}}\right]^{T}=\left[\dot{\varphi}_{1}, \dot{\varphi}_{2}, \dot{\varphi}_{3}\right]^{T},
$$

where $\omega_{v_{1}}, \omega_{v_{2}}$ and $\omega_{v_{3}}$ are the angular velocities of the robot wheels. From the works of Djebrani et al. (2011; 2010b; 2009), Mourioux et al. (2006) and Poisson et al. (2001), the Jacobian $J$ can be obtained, providing a direct relation between global velocities and angular velocities of the wheels:

$$
\begin{gathered}
\omega_{v}=\frac{1}{r} J \dot{\xi}_{p}, \\
\left\{\begin{array}{l}
\dot{x} \sin \left(\vartheta+\alpha_{1}\right)-\dot{y} \cos \left(\vartheta+\alpha_{1}\right)-\dot{\vartheta} R_{1}=r \omega_{v_{1}}, \\
\dot{x} \sin \left(\vartheta+\alpha_{2}\right)-\dot{y} \cos \left(\vartheta+\alpha_{2}\right)-\dot{\vartheta} R_{2}=r \omega_{v_{2}}, \\
\dot{x} \sin \left(\vartheta+\alpha_{3}\right)-\dot{y} \cos \left(\vartheta+\alpha_{3}\right)-\dot{\vartheta} R_{3}=r \omega_{v_{3}},
\end{array}\right.
\end{gathered}
$$

where $J=A R(\vartheta)$,

$$
A=\left[\begin{array}{lll}
\sin \alpha_{1} & -\cos \alpha_{1} & -R_{1} \\
\sin \alpha_{2} & -\cos \alpha_{2} & -R_{2} \\
\sin \alpha_{3} & -\cos \alpha_{3} & -R_{3}
\end{array}\right]
$$

and

$$
R(\vartheta)=\left[\begin{array}{ccc}
\cos \vartheta & \sin \vartheta & 0 \\
-\sin \vartheta & \cos \vartheta & 0 \\
0 & 0 & 1
\end{array}\right]
$$

For each axle, we set $R_{i}$ as the distance $O^{\prime} O_{i}$ (the variable distance determined by the sphere of the $i$-th axle in contact with the floor):

$$
\begin{aligned}
& R_{i} \\
& =\left\{\begin{array}{c}
R_{i_{\min }}+\frac{1}{2} \Delta R \text { if } 0 \leq \varphi_{i}<\frac{\pi}{2} \text { and } \pi \leq \varphi_{i}<\frac{3 \pi}{2}, \\
R_{i_{\max }}-\frac{1}{2} \Delta R \text { if } \frac{\pi}{2} \leq \varphi_{i}<\pi \text { and } \frac{3 \pi}{2} \leq \varphi_{i}<2 \pi,
\end{array}\right.
\end{aligned}
$$

where $\Delta R=R_{i_{\max }}-R_{i_{\min }}$ corresponds to the difference of the radii between the two truncated spheres of the same axle, $R_{i_{\max }}$ and $R_{i_{\min }}$ characterize the configuration of the wheels under the axle, $\varphi_{i}$ is the angular coordinate of the $i$-th axle. Now we are ready to develop the dynamic model.

4.2. Dynamic modelling. Let us first determine the motor dynamics. The shaft output torque is determined by taking into account all forces acting on each wheel. The main force acting on the wheels is the force to overcome the inertia of the base while accelerating. This force consists of two components: the first force accelerates the base laterally while the second accelerates angularly. Due to the inertia of the base, the force needs to be applied to accelerate and decelerate the base. This force is exerted by the wheels, which transfer the motor torque to the drive surface. The required force can be calculated using Newton's second law (Spong et al., 1989):

$$
F=D \ddot{\xi}_{p},
$$

where

$$
D=\left[\begin{array}{ccc}
m_{R} & 0 & 0 \\
0 & m_{R} & 0 \\
0 & 0 & I_{R}
\end{array}\right] .
$$

Using Eqn. (8) with $J=A R(\vartheta)$, we obtain

$$
\ddot{\xi}_{p}=r \dot{R}^{T} A^{-1} \omega_{v}+r R^{T} A^{-1} \dot{\omega}_{v} .
$$

The magnitude of the global forces $F_{x}, F_{y}$ and the moment $M_{t}$ can be written using the base mass $m_{R}$ and the moment of inertia $I_{R}$ as

$$
\left[\begin{array}{l}
F_{x} \\
F_{y} \\
M_{t}
\end{array}\right]=\left[\begin{array}{ccc}
m_{R} & 0 & 0 \\
0 & m_{R} & 0 \\
0 & 0 & I_{R}
\end{array}\right]\left[\begin{array}{l}
\ddot{x} \\
\ddot{y} \\
\ddot{\vartheta}
\end{array}\right] .
$$

The global forces $F_{x}$ and $F_{y}$ can be substituted by the sum of the lateral shaft force components in the $x$ and $y$ directions, respectively, while the global moment $M_{t}$ is given by the product of the combined local force vectors and the radius on which they act (Fig. 7):

$$
\left\{\begin{aligned}
F_{x}= & f_{1_{x}}+f_{2_{x}}+f_{3_{x}} \\
= & -f_{1} \sin \left(\vartheta+\alpha_{1}\right)-f_{2} \sin \left(\vartheta+\alpha_{2}\right) \\
& -f_{3} \sin \left(\vartheta+\alpha_{3}\right), \\
F_{y}= & f_{1_{y}}+f_{2_{y}}+f_{3_{y}} \\
& +f_{1} \cos \left(\vartheta+\alpha_{1}\right)+f_{2} \cos \left(\vartheta+\alpha_{2}\right) \\
& +f_{3} \cos \left(\vartheta+\alpha_{3}\right), \\
M_{t}= & f_{1} R_{1}+f_{2} R_{2}+f_{3} R_{3},
\end{aligned}\right.
$$

or, in a more compact form,

$$
F=\left[m_{R} \ddot{x}, m_{R} \ddot{y}, I_{R} \ddot{\vartheta}\right]^{T}=J^{T}\left[f_{1}, f_{2}, f_{3}\right]^{T} .
$$

We can write

$$
\left[f_{1}, f_{2}, f_{3}\right]^{T}=J^{-T}\left[m_{R} \ddot{x}, m_{R} \ddot{y}, I_{R} \ddot{\vartheta}\right]^{T},
$$




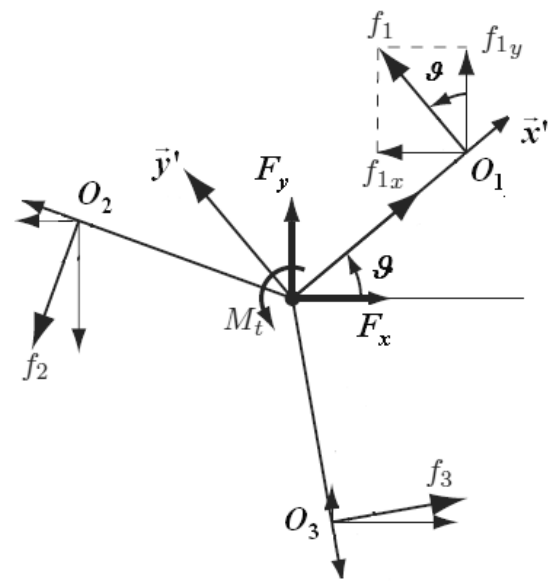

Fig. 7. Dynamic diagram of the three-wheel base.

where $f_{i}$ is the traction force of each wheel. By replacing (15) in (19), we obtain

$$
f=A^{-T} R D\left[r \dot{R}^{T} A^{-1} \omega_{v}+r R^{T} A^{-1} \dot{\omega}_{v}\right] .
$$

The dynamics of each wheel driven by a DC motor can be described as

$$
\begin{aligned}
I_{m} \dot{\omega}_{m_{i}}+\left(\frac{C_{m} C_{e}}{R_{a}}+b_{m}\right) \omega_{m_{i}}-\frac{C_{m}}{R_{a}} \tau_{v_{i}} \\
=-\frac{r}{n_{m}} f_{i},
\end{aligned}
$$

where $I_{m}$ is the combined moment of inertia of the motor, gear train and wheel referred to the motor shaft, $\omega_{m_{i}}$ is the rotational speed of the motor shaft, $R_{a}$ is the armature resistance, $C_{e}$ is the Electro-Motive Force (EMF) constant, $C_{m}$ is the motor torque constant, $b_{m}$ is the viscous friction coefficient which is a combination of the motor and gear trains, $n_{m}$ is the gear ratio, $\tau_{v_{i}}$ is the armature voltage applied (specAmotor, 2011).

In matrix form we have to consider the relation $\omega_{m_{i}}=n_{m} \omega_{v_{i}}$ between the motor and robot rotational speeds:

$$
f=-P \dot{\omega}_{v}-Q \omega_{v}+E_{v} \tau_{v}
$$

where

$$
\begin{aligned}
P & =\frac{n_{m}^{2}}{r} I_{m}\left[\begin{array}{lll}
1 & 0 & 0 \\
0 & 1 & 0 \\
0 & 0 & 1
\end{array}\right], \\
Q & =\frac{n_{m}^{2}}{r}\left(\frac{C_{m} C_{e}}{R_{a}}+b_{m}\right)\left[\begin{array}{lll}
1 & 0 & 0 \\
0 & 1 & 0 \\
0 & 0 & 1
\end{array}\right], \\
E_{v} & =\frac{n_{m}}{r} \frac{C_{m}}{R_{a}}\left[\begin{array}{lll}
1 & 0 & 0 \\
0 & 1 & 0 \\
0 & 0 & 1
\end{array}\right] .
\end{aligned}
$$

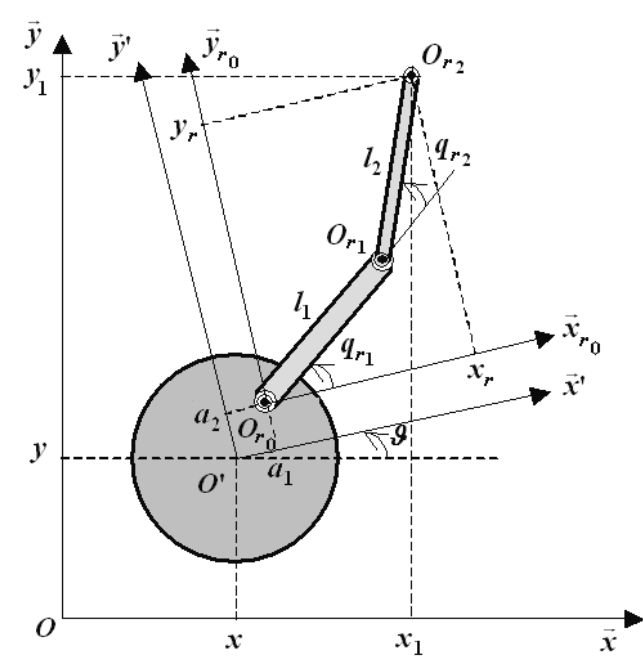

Fig. 8. Planar mobile manipulator with an omnidirectional platform.

By replacing (20) in (22), one can write the dynamical equation of the mobile robot as

$$
M_{v_{1}}\left(\xi_{p}\right) \dot{\omega}_{v}+C_{v_{1}}\left(\xi_{p}, \omega_{v}\right) \omega_{v}=E_{v} \tau_{v}
$$

where

$$
\begin{gathered}
M_{v_{1}}\left(\xi_{p}\right)=\left(r A^{-T} R D R^{T} A^{-1}+P\right), \\
C_{v_{1}}\left(\xi_{p}, \omega_{v}\right)=\left(r A^{-T} R D \dot{R}^{T} A^{-1}+Q\right)
\end{gathered}
$$

are the inertia and coupling matrices, respectively.

\section{Omnidirectional mobile manipulator modelling}

Consider the mobile manipulator depicted in Fig. 8. It consists of an omnidirectional mobile platform and a twolink manipulator. The platform moves by driving the three wheels. The manipulator is constructed as a two-link planar arm with motors attached to the joints.

5.1. Kinematic modelling. We reduce the location to the planar position of the end-effector in the horizontal plane. The location of the platform is given by a vector $\xi_{p}=[x, y, \vartheta]^{T}$ which defines the position and the orientation of the platform in the frame $\mathcal{R}$. The position of the point $O_{r_{2}}$ in the frame $\mathcal{R}$ is thus given by a vector $\xi=\left[x_{1}, y_{1}\right]^{T}$. The coordinates of $O_{r_{0}}$ in $\mathcal{R}^{\prime}$ are given by $\left[a_{1}, a_{2}\right]^{T}$ (see Fig. 8). According to (1), the KM of this 
mobile manipulator is (Bayle, 2001; Campion et al., 1996)

$$
\left\{\begin{aligned}
x_{1}= & {\left[a_{1}+l_{1} \cos \left(q_{r_{1}}\right)+l_{2} \cos \left(q_{r_{1}}+q_{r_{2}}\right)\right] \cos (\vartheta) } \\
& -\left[a_{2}+l_{1} \sin \left(q_{r_{1}}\right)+l_{2} \sin \left(q_{r_{1}}+q_{r_{2}}\right)\right] \\
& \sin (\vartheta)+x, \\
y_{1}= & {\left[a_{1}+l_{1} \cos \left(q_{r_{1}}\right)+l_{2} \cos \left(q_{r_{1}}+q_{r_{2}}\right)\right] \sin (\vartheta) } \\
& +\left[a_{2}+l_{1} \sin \left(q_{r_{1}}\right)+l_{2} \sin \left(q_{r_{1}}+q_{r_{2}}\right)\right] \\
& \cos (\vartheta)+y .
\end{aligned}\right.
$$

From (24), we get the ILKM (Instantaneous Location Kinematic Model):

$$
\begin{aligned}
& \dot{\xi}= {\left[\begin{array}{c}
\dot{x}_{1} \\
\dot{y}_{1}
\end{array}\right]=\bar{J}\left(q_{r_{1}}, q_{r_{2}}, \vartheta\right)\left[\begin{array}{c}
\dot{q}_{r_{1}} \\
\dot{q}_{r_{2}} \\
\dot{x} \\
\dot{y} \\
\dot{\vartheta}
\end{array}\right], } \\
& \\
& \bar{J}\left(q_{r_{1}}, q_{r_{2}}, \vartheta\right) \\
&=\left[\begin{array}{llll}
-l_{1} S_{1 \vartheta}-l_{2} S_{12 \vartheta} & -l_{2} S_{12 \vartheta} & 1 & 0 \\
l_{1} C_{1 \vartheta}+l_{2} C_{12 \vartheta} & l_{2} C_{12 \vartheta} & 0 & 1 \\
& -a_{1} S_{\vartheta}-a_{2} C_{\vartheta}-l_{1} S_{1 \vartheta}-l_{2} S_{12 \vartheta} \\
& +a_{1} C_{\vartheta}-a_{2} S_{\vartheta}+l_{1} C_{1 \vartheta}+l_{2} C_{12 \vartheta}
\end{array}\right]
\end{aligned}
$$

with the following intermediate variables:

$$
\begin{aligned}
C_{1 \vartheta} & =\cos \left(q_{r_{1}}+\vartheta\right), \\
S_{1 \vartheta} & =\sin \left(q_{r_{1}}+\vartheta\right), \\
C_{12 \vartheta} & =\cos \left(q_{r_{1}}+q_{r_{2}}+\vartheta\right), \\
S_{12 \vartheta} & =\sin \left(q_{r_{1}}+q_{r_{2}}+\vartheta\right), \\
C_{\vartheta} & =\cos (\vartheta), \\
S_{\vartheta} & =\sin (\vartheta) .
\end{aligned}
$$

5.2. Dynamic modelling. A mobile manipulator dynamic equation can be obtained using the Lagrangian approach (Yamamoto, 1994; Liu and Lewis, 1990). It is in the form

$$
M(q) \dot{\omega}+C(q, \omega)=\tau
$$

where $q=\left[q_{r}, \xi_{p}\right]^{T}, \omega=\left[\omega_{r}, \omega_{v}\right]^{T}, \dot{\omega}=\left[\dot{\omega}_{r}, \dot{\omega}_{v}\right]^{T}, \tau=$ $\left[\tau_{r}, E_{v} \tau_{v}\right]^{T}$.

The dynamic model of the manipulator (26), can be expressed in terms of the dynamic interaction and coupling. The motion equation of the manipulator subject to the vehicle motion is given in the following form
(Djebrani et al., 2011):

$$
\left\{\begin{array}{l}
M_{r}\left(q_{r}\right) \dot{\omega}_{r}+C_{r_{1}}\left(q_{r}, \omega_{r}\right) \omega_{r}+C_{r_{2}}\left(q_{r}, \omega_{r}, \omega_{v}\right) \\
=\tau_{r}-R_{r}\left(q_{r}, \xi_{p}\right) \dot{\omega}_{v} \\
C_{r}\left(q_{r}, \omega_{r}, \omega_{v}\right) \\
=C_{r_{1}}\left(q_{r}, \omega_{r}\right) \omega_{r}+C_{r_{2}}\left(q_{r}, \omega_{r}, \omega_{v}\right),
\end{array}\right.
$$

where $M_{r}$ and $C_{r_{1}}$ are the inertia matrix and the Coriolis and centrifugal terms of the manipulator given by Eqn. (3), $C_{r_{2}}$ denotes the Coriolis and centrifugal terms caused by the angular motion of the platform, $\tau_{r}$ is the input torque/force for the manipulator and $R_{r}$ is the inertia matrix which represents the effect of the vehicle dynamics on the manipulator. We note that $C_{r_{2}}$ and $R_{r}$ are the terms added to the equation of motion of the manipulator. They represent the dynamic interaction caused by the motion of the mobile platform.

The motion equation of the platform retains the following form:

$$
\left\{\begin{array}{l}
M_{v_{1}}\left(\xi_{p}\right) \dot{\omega}_{v}+C_{v_{1}}\left(\xi_{p}, \omega_{v}\right) \omega_{v}+C_{v_{2}}\left(q_{r}, \xi_{p}, \omega_{r}, \omega_{v}\right) \\
=E_{v} \tau_{v}-M_{v_{2}}\left(q_{r}, \xi_{p}\right) \dot{\omega}_{v}-R_{v}\left(q_{r}, \xi_{p}\right) \dot{\omega}_{r}, \\
C_{v}\left(q_{r}, \xi_{p}, \omega_{r}, \omega_{v}\right) \\
\quad=C_{v_{1}}\left(\xi_{p}, \omega_{v}\right) \omega_{v}+C_{v_{2}}\left(q_{r}, \xi_{p}, \omega_{r}, \omega_{v}\right), \\
M_{v}\left(q_{r}, \xi_{p}\right)=M_{v_{1}}\left(\xi_{p}\right)+M_{v_{2}}\left(q_{r}, \xi_{p}\right),
\end{array}\right.
$$

where $M_{v_{1}}$ and $C_{v_{1}}$ are the mass inertia matrix and the velocity dependent terms of the platform which are defined in Eqn. (23), $M_{v_{2}}$ and $C_{v_{2}}$ represent the inertial term as well as the Coriolis and centrifugal terms due to the manipulator presence, $E_{v} \tau_{v}$ is the input torque to the vehicle, $E_{v}$ is a constant matrix and $R_{v}$ represents the inertia matrix which reflects the dynamic effect of the arm motion on the vehicle.

The dynamic model (Eqn. (26)) of the mobile manipulator is described by

$$
\left[\begin{array}{ll}
M_{r} & R_{r} \\
R_{v} & M_{v}
\end{array}\right]\left[\begin{array}{c}
\dot{\omega}_{r} \\
\dot{\omega}_{v}
\end{array}\right]+\left[\begin{array}{c}
C_{r} \\
C_{v}
\end{array}\right]=\left[\begin{array}{c}
\tau_{r} \\
E_{v} \tau_{v}
\end{array}\right] .
$$

In order to write a global control law, let us consider

$$
\begin{aligned}
X & =\left[x_{1}, x_{2}, x_{3}, x_{4}, x_{5}, x_{6}, x_{7}, x_{8}, x_{9}, x_{10}\right]^{T} \\
& =\left[q_{r_{1}}, q_{r_{2}}, x, y, \vartheta, \omega_{r_{1}}, \omega_{r_{2}}, \omega_{v_{1}}, \omega_{v_{2}}, \omega_{v_{3}}\right]^{T}
\end{aligned}
$$

as the state vector of the mobile manipulator. The state space equation is then given by (Djebrani et al., 2011; 2010b)

$$
\dot{X}=f(X)+g(X) \tau,
$$

where $f(X)$ and $g(X)$ are smooth vector fields on $\mathbb{R}^{n}$ :

$$
f(X)=\left[\begin{array}{c}
\omega_{r} \\
r J\left(\xi_{p}\right)^{-1} \omega_{v} \\
-M^{-1} C
\end{array}\right]
$$




$$
\begin{aligned}
& f(X)=\left\{\begin{aligned}
& f_{1}(X)= x_{6} \\
& f_{2}(X)= x_{7} \\
& f_{3}(X)= \frac{2}{3} r x_{8} \sin x_{5} \\
& \quad+r\left(\frac{1}{\sqrt{3}} \cos x_{5}-\frac{1}{3} \sin x_{5}\right) x_{9} \\
& \quad+r\left(-\frac{1}{\sqrt{3}} \cos x_{5}-\frac{1}{3} \sin x_{5}\right) x_{10}, \\
& f_{4}(X)=-\frac{2}{3} r x_{8} \cos x_{5} \\
& \quad+r\left(\frac{1}{\sqrt{3}} \sin x_{5}+\frac{1}{3} \cos x_{5}\right) x_{9} \\
& \quad+r\left(-\frac{1}{\sqrt{3}} \sin x_{5}+\frac{1}{3} \cos x_{5}\right) x_{10}, \\
& f_{5}(X)=-H r x_{8}-H r x_{9}-H r x_{10}, \\
& {\left[f_{6}(X), f_{7}(X), f_{8}(X), f_{9}(X), f_{10}(X)\right]^{T} } \\
&=-M^{-1} C,
\end{aligned}\right. \\
& g(X)=\left[\begin{array}{l}
0_{5 \times 5} \\
M^{-1}
\end{array}\right]
\end{aligned}
$$

From Eqns. (32) and (33) it is clear that $f(X)$ and $g(X)$ depend only on the state variable $X$. The parameter $H$ is a constant. In the next section we will propose a new feedback control law of our mobile manipulator.

\section{Local coordinate linearization}

We consider the nonlinear multivariable system as described in state space form by the following equations:

$$
\left\{\begin{array}{l}
\dot{X}=f(X)+\sum_{j=1}^{5} g_{j}(X) \tau_{j}, \\
y_{j}=h_{j}(X), \quad j=1,2,3,4,5 .
\end{array}\right.
$$

According to Isidori (1995) we can recall the conditions for the solvability of a MIMO state space exact linearization problem as follows.

Proposition 1. Suppose the matrix $g\left(X^{o}\right)$ has rank $m$. Then, the state space exact linearization problem is solvable if and only if

(i) for each $0 \leq i \leq n-1$, the distribution

$$
G_{i}=\operatorname{span}\left\{\operatorname{ad}_{f}^{k} g_{j}: 0 \leq k \leq i, 1 \leq j \leq m\right\}
$$

has constant dimension near $X^{o}$ (equilibrium point);

(ii) the distribution $G_{n-1}$ has dimension n;

(iii) for each $0 \leq i \leq n-2$, the distribution $G_{i}$ is involutive.

Theorem 1. The change of variables allows linearization of the system (30):

$$
\left\{\begin{aligned}
x_{1}= & z_{1}, \\
x_{2}= & z_{3}, \\
x_{3}= & z_{5}, \\
x_{4}= & z_{7}, \\
x_{5}= & z_{9}, \\
x_{6}= & z_{2}, \\
x_{7}= & z_{4}, \\
x_{8}= & \frac{1}{r} z_{6} \sin z_{9}-\frac{1}{r} z_{8} \cos z_{9}-\frac{1}{r} R_{1} z_{10}, \\
x_{9}= & \frac{1}{r}\left(\frac{\sqrt{3}}{2} \cos z_{9}-\frac{1}{2} \sin z_{9}\right) z_{6}, \\
& +\frac{1}{r}\left(\frac{\sqrt{3}}{2} \sin z_{9}+\frac{1}{2} \cos z_{9}\right) z_{8}-\frac{1}{r} R_{2} z_{10}, \\
& \quad \frac{1}{r}\left(\frac{-\sqrt{3}}{2} \cos z_{9}-\frac{1}{2} \sin z_{9}\right) z_{6}, \\
& \quad+\frac{1}{r}\left(-\frac{\sqrt{3}}{2} \sin z_{9}+\frac{1}{2} \cos z_{9}\right) z_{8}-\frac{1}{r} R_{3} z_{10}
\end{aligned}\right.
$$

Proof. In our case we have to find five functions $h_{1}(X)$, $h_{2}(X), h_{3}(X), h_{4}(X), h_{5}(X)$ such that

$$
L_{g_{j}} L_{f}^{k} h_{j}(X)=0
$$

for all $0 \leq k \leq r_{j}-2,1 \leq j \leq m$. The relative degrees $r_{j}$ have to fulfill the condition $r_{1}+r_{2}+r_{3}+r_{4}+r_{5}=n$. In our case the distribution $G_{o}=\operatorname{span}\left\{g_{1}, g_{2}, g_{3}, g_{4}, g_{5}\right\}$ has dimension $m=5$. Moreover, since $\left[g_{i}, g_{j}\right]=0$ for all $i, j \in\{1,2,3,4,5\}$, we see that the distribution in question is involutive.

The distribution has maximal dimension $n=10$, which is the dimension of the state vector. We see that $\operatorname{dim}\left(G_{i}\right)=10$ for $i \in[1,9]$ and $G_{j}$ for $j \in[1,8]$ are trivially involutive. The system satisfies the hypotheses of the proposition. In order to solve the full state linearization problem, we have to find functions $h_{j}(x)$ that check the condition (36). It is easy to conclude that we must have

$$
\frac{\partial h_{j}}{\partial x_{6}}=\frac{\partial h_{j}}{\partial x_{7}}=\frac{\partial h_{j}}{\partial x_{8}}=\frac{\partial h_{j}}{\partial x_{9}}=\frac{\partial h_{j}}{\partial x_{10}}=0
$$

for $j=1,2,3,4,5$. One can choose $h_{1}(X)=x_{1}$, $h_{2}(X)=x_{2}, h_{3}(X)=x_{3}, h_{4}(X)=x_{4}, h_{5}(X)=x_{5}$. Let us analyse the relative degree of the system. The system has a vector relative degree $\left\{r_{1}, r_{2}, r_{3}, r_{4}, r_{5}\right\}$. One can check that $L_{g_{1}} h_{1}(X)=L_{g_{2}} h_{2}(X)=L_{g_{3}} h_{3}(X)=$ $L_{g_{4}} h_{4}(X)=L_{g_{5}} h_{5}(X)=0$.

The relative degrees are $r_{1}=r_{2}=r_{3}=r_{4}=r_{5}=$ 2. Then we can check that the following matrix is not 
singular:

$$
\alpha(X)=\left[\begin{array}{ccc}
L_{g_{1}} L_{f} h_{1}(X) & \ldots & L_{g_{5}} L_{f} h_{1}(X) \\
\vdots & \ddots & \vdots \\
L_{g_{1}} L_{f} h_{5}(X) & \ldots & L_{g_{5}} L_{f} h_{5}(X)
\end{array}\right]
$$

Then the smooth functions that define the local change of variables that linearize the system are

$$
\left\{\begin{array}{l}
z_{1}=h_{1}(X)=x_{1}, \\
z_{2}=L_{f} h_{1}(X), \\
z_{3}=h_{2}(X)=x_{2}, \\
z_{4}=L_{f} h_{2}(X), \\
z_{5}=h_{3}(X)=x_{3}, \\
z_{6}=L_{f} h_{3}(X), \\
z_{7}=h_{4}(X)=x_{4}, \\
z_{8}=L_{f} h_{4}(X), \\
z_{9}=h_{5}(X)=x_{5}, \\
z_{10}=L_{f} h_{5}(X),
\end{array}\right.
$$

where

$$
\left\{\begin{aligned}
L_{f} h_{1}(X)= & x_{6}, \\
L_{f} h_{2}(X)= & x_{7}, \\
L_{f} h_{3}(X)= & \frac{2}{3} r x_{8} \sin x_{5} \\
& +r\left(\frac{1}{\sqrt{3}} \cos x_{5}-\frac{1}{3} \sin x_{5}\right) x_{9} \\
& +r\left(-\frac{1}{\sqrt{3}} \cos x_{5}-\frac{1}{3} \sin x_{5}\right) x_{10}, \\
L_{f} h_{4}(X)= & -\frac{2}{3} r x_{8} \cos x_{5} \\
& +r\left(\frac{1}{\sqrt{3}} \sin x_{5}+\frac{1}{3} \cos x_{5}\right) x_{9} \\
& +r\left(-\frac{1}{\sqrt{3}} \sin x_{5}+\frac{1}{3} \cos x_{5}\right) x_{10}, \\
L_{f} h_{5}(X)= & -H r x_{8}-H r x_{9}-H r x_{10} .
\end{aligned}\right.
$$

The control law $\tau$ is written as follows:

$$
\tau=\left[\begin{array}{c}
\tau_{1} \\
\tau_{2} \\
\tau_{3} \\
\tau_{4} \\
\tau_{5}
\end{array}\right]=\alpha^{-1}(X)\left(-\beta(X)+\left[\begin{array}{l}
v_{1} \\
v_{2} \\
v_{3} \\
v_{4} \\
v_{5}
\end{array}\right]\right),
$$

where

$$
\beta(X)=L_{f}^{2} h_{j}(X), \quad j=1,2,3,4,5,
$$

and

$$
\left[\begin{array}{c}
\dot{z}_{1} \\
\dot{z}_{3} \\
\dot{z}_{5} \\
\dot{z}_{7} \\
\dot{z}_{9}
\end{array}\right]=\left[\begin{array}{c}
z_{2} \\
z_{4} \\
z_{6} \\
z_{8} \\
z_{10}
\end{array}\right]
$$

$$
\left[\begin{array}{c}
\dot{z}_{2} \\
\dot{z}_{4} \\
\dot{z}_{6} \\
\dot{z}_{8} \\
\dot{z}_{10}
\end{array}\right]=\left[\begin{array}{c}
v_{1} \\
v_{2} \\
v_{3} \\
v_{4} \\
v_{5}
\end{array}\right]
$$

where $v$ is a new input to be determined. We obtain a simple relationship between the output $q$ and the new input $v$ :

$$
v=\ddot{q}=\ddot{q}^{*}+K_{\mathrm{der}} \dot{\tilde{q}}+K_{\text {pro }} \tilde{q},
$$

where $\tilde{q}=q^{*}-q, K_{\text {der }}$ and $K_{\text {pro }}$ are positive definite matrices. After linearization, one can consider an outer loop impedance regulation which can be used to drive the robot between obstacles or to collaborate with other robots.

\section{Virtual impedance control strategy}

The control objective requires multiple goals, such as reaching targets, or avoiding static and dynamic obstacles. In order to solve the above-mentioned problems, we propose a control strategy based on a dynamic structure of the robot and the impedance control technique. This model was proposed by Arai and Ota (1996) in order to generate only forces. In our work we will use that concept to generate forces in a low level outer loop. It allows us to modify the robot trajectory in terms of the imposed impedance $Z_{d}$. This technique determines the motion of a robot by means of a desired trajectory $q^{*}$ modified by a sum of different forces (Goldenberg, 1988; Hogan, 1985). The closed loop dynamical equation for our robot must be expressed as

$$
\begin{array}{r}
M_{d}\left(\ddot{q}^{*}-\ddot{q}\right)+B_{d}\left(\dot{q}^{*}-\dot{q}\right)+K_{d}\left(q^{*}-q\right) \\
=-F_{\text {ext }}
\end{array}
$$

where $F_{\text {ext }}=\sum_{i} F_{\mathrm{obs}_{i}}$ represents all the forces exerted on the robot, $F_{\mathrm{obs}_{i}}$ is the force which avoids the $i$-th obstacle. $Z_{d}=M_{d} p^{2}+B_{d} p+K_{d}$ is the desired impedance, $M_{d}, B_{d}, K_{d}$ are diagonal positive definite matrices of desired mass, damping and spring impedance effects, $p \equiv \mathrm{d} / \mathrm{d} t$. Equation (44) can be expressed in terms of the desired impedance and the trajectory tracking as

$$
e_{d}=\left(q^{*}-q\right)+\frac{F_{\mathrm{ext}}}{Z_{d}}
$$

where $e_{d}$ is a new auxiliary signal error $e_{d}=q_{d}^{*}-q$. If $e_{d} \rightarrow 0$, then Eqn. (44) is realized. The new desired trajectory $q_{d}^{*}$ can be seen as the sum of the desired trajectory $q^{*}$ and the force correction $F_{\text {ext }} / Z_{d}$.

The control law given by Eqn. (39) is then modified to take into account the presence of obstacles. So the new desired trajectory is given by $q_{d}^{*}(t)=q^{*}(t)+F_{\text {ext }} / Z_{d}$ and the $v$ signal from Eqn. (43) by

$$
v=\ddot{q}_{d}^{*}+K_{\mathrm{der}}\left(\dot{q}_{d}^{*}-\dot{q}\right)+K_{\mathrm{pro}}\left(q_{d}^{*}-q\right) .
$$


The magnitude $F_{\text {obs }}$ is obtained as (Carelli et al., 1999; Borenstein and Koren, 1991)

$$
F_{\mathrm{obs}}=a_{F_{\mathrm{obs}}}-b_{F_{\mathrm{obs}}}\left(d_{\mathrm{obs}}(t)-d_{\mathrm{min}}\right)^{2} .
$$

where $a_{F_{\mathrm{obs}}}$ and $b_{F_{\mathrm{obs}}}$ are positive constants satisfying the condition

$$
a_{F_{\mathrm{obs}}}=b_{F_{\mathrm{obs}}}\left(d_{\max }-d_{\mathrm{min}}\right)^{2},
$$

$d_{\max }$ being the maximum distance between the robot and the detected obstacle that causes a nonzero repulsive force, $d_{\text {min }}$ represents the minimum distance accepted between the robot and the obstacle, and $d_{\mathrm{obs}}(t)$ is the distance measured between the robot and the obstacle $d_{\text {min }}<d_{\text {obs }}(t)<d_{\max }$ (see Fig. 9). Note that the bound

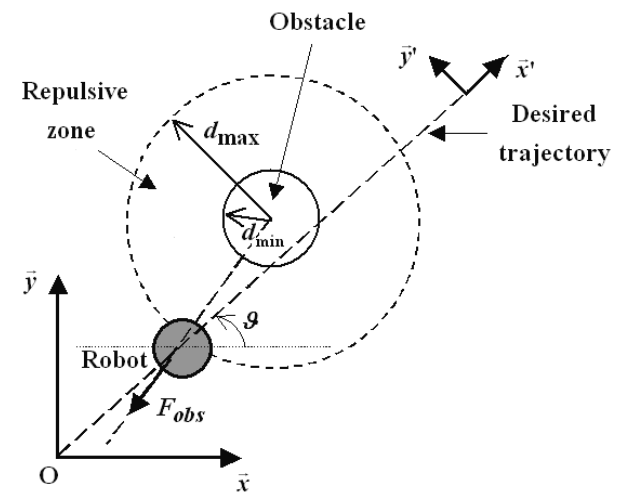

Fig. 9. Repulsive force caused by an obstacle.

$d_{\text {max }}$ characterizes the repulsion zone, which is the region inside which the repulsion force has a non-zero value.

As mentioned in Section 2, we introduce an adaptive fuzzy algorithm as an intelligent control solution to select the desired behavior $Z_{d}$ (Abdessemed et al., 2004; Abdessemed and Benmahammed, 2001). Figure 10 gives a schematic block diagram of this architecture. In this figure one can notice that the inputs to the fuzzy controller are the generated virtual forces $\left(F_{o b s}\right)$ and measured distances named $d_{4}$ and $d_{5}$. These distances $d_{4}$ and $d_{5}$ concern the distances measured with the lateral sensors. Figure 11 shows the impedance fuzzy sets associated with the generated forces and Fig. 12 presents the membership functions of $d_{4}$ and $d_{5}$. Each membership function is a triangular-shaped membership function with the collection of linguistic values: $\{V L, L, M, H, V H\}$. The meaning of each linguistic value should be clear from its mnemonics; for example, $V L$ stands for verylow, $L$ for low, $M$ for middle, $H$ for high and $V H$ for veryhigh.

Let us denote the fuzzy block as a three input-single output controller. We construct five numerical values for the desired behavior $Z_{d},\{V S, S, M, B, V B\}$. The meaning of each linguistic value, $V S$ stands for verysmall, $S$ for small, $M$ for middle, $B$ for big and $V B$ for verybig.

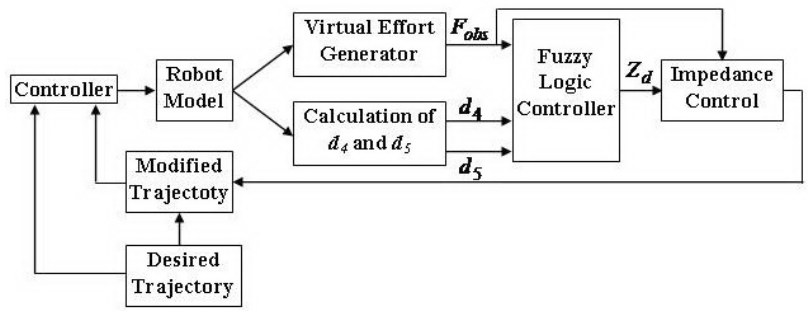

Fig. 10. Block diagram of the controlled system.

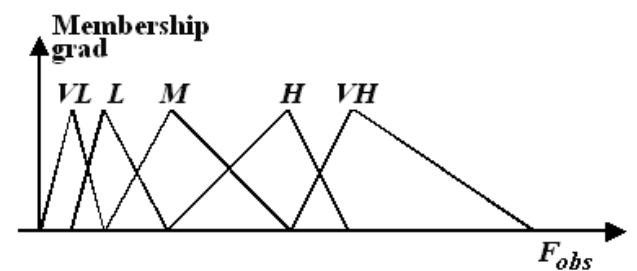

Fig. 11. Membership functions of input $F_{o b s}$.

Consequently, simple robot impedance generation can be described as the following linguistic rules:

Rule 1 : IF $F_{\text {obs }}$ is $V L$ and $d_{4}$ is $V H$ and $d_{5}$ is $V H$ THEN $Z_{d_{1}}$ is $V S$

Rule 2 : IF $F_{\text {obs }}$ is $L$ and $d_{4}$ is $V H$ and $d_{5}$ is $V H$ THEN $Z_{d_{2}}$ is $S$

Rule 3: IF $F_{\text {obs }}$ is $M$ and $d_{4}$ is $V H$ and $d_{5}$ is $V H$ THEN $Z_{d_{3}}$ is $M$

Rule 4 : IF $F_{\text {obs }}$ is $H$ and $d_{4}$ is $V L$ and $d_{5}$ is $V L$ THEN $Z_{d_{4}}$ is $B$

Rule 5 : etc.

such that $F_{\text {obs }}, d_{4}$ and $d_{5}$ are the inputs and $Z_{d}$ is the output. Based on the above rules, the Sugeno defuzzifier strategy is chosen as described by Sugeno and nishida (1985) in order to derive $Z_{d}$ as the output. We defuzzify the membership function using min-operation implication, and we have

$$
Z_{d}=\frac{\sum_{l=1}^{n_{\text {rules }}} \mu_{l} Z_{d_{l}}}{\sum_{l=1}^{n_{\text {rules }}} \mu_{l}},
$$

$l=1,2, \ldots, n_{\text {rules }}, n_{\text {rules }}$ being the number of rules, $Z_{d_{l}}$

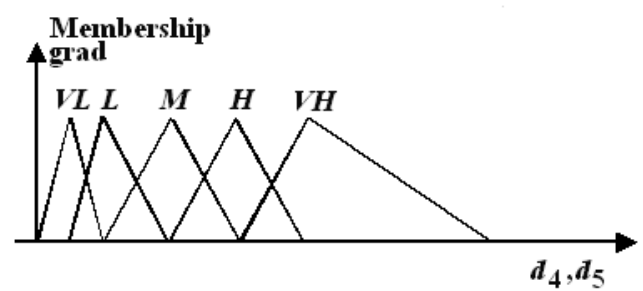

Fig. 12. Membership functions of inputs $d_{4}$ and $d_{5}$. 
the rule's consequent of the $l$-th rule whereas $\mu_{l}$ is the corresponding degree of fulfillment for the $l$-th rule.

\section{Simulation results}

Our simulations were realized on a model of the omnidirectional mobile manipulator. Its description and modelling were presented in Section 5 with $a_{1}=a_{2}=0$. All the simulations experiments were carried out by considering a set of physical parameters for the dynamic model of the omnidirectional mobile manipulator given by the mass link $1 m_{1}=10 \mathrm{~kg}$, the mass link $2 m_{2}=5 \mathrm{~kg}$, the inertia link $1 I_{1}=0.05 \mathrm{~kg} \cdot \mathrm{m}^{2}$, the inertia link $2 I_{2}=$ $0.025 \mathrm{~kg} \cdot \mathrm{m}^{2}$, the length link $l_{1}=l_{2}=0.5 \mathrm{~m}$, the base mass $m_{R}=20 \mathrm{~kg}$, the radius of the platform $R=0.3 \mathrm{~m}$, the moment of inertia of the platform $I_{R}=0.9 \mathrm{~kg} \cdot \mathrm{m}^{2}$, the moment of inertia of the motor $I_{m}=1.380 e-5 \mathrm{~kg} . \mathrm{m}^{2}$, the armature resistance $R_{a}=0.317 \Omega$, the viscous friction coefficient $b_{m}=0.004 \mathrm{~N} \cdot \mathrm{m}$, the electromotive force constant $C_{e}=3.02 e-2 \mathrm{~V} \cdot \mathrm{s} / \mathrm{rad}$, the motor torque constant $C_{m}=3.02 e-2 \mathrm{~N} \cdot \mathrm{m} / \mathrm{A}$, the radius of the sphere $r=0.03 \mathrm{~m}, R_{i_{\max }}=0.2 \mathrm{~m}$ and $R_{i_{\min }}=0.13 \mathrm{~m}$.

The simulation results are shown in Figs. 13-17, and indicate the successful control operation for the input trajectories imposed for the base from $t_{0}=0: x^{*}(t)=$ $0.05 \sin (1.6 t+0.2)+0.09 \sin (2 t+0.15)$ and $y^{*}(t)=$ $0.08 \sin (2.6 t+0.02)+0.02 \sin (1.2 t+0.35)$, the articular movement imposed for the arm is $q_{r}^{*}=\left[q_{r_{1}}^{*}, q_{r_{2}}^{*}\right]^{T}=$ $[0.1 \sin (2 t+0.1), 0.2 \cos (t+0.1)]^{T}$. Based on the evaluation method of the obstacles configuration from the information of the sensors, the mobile manipulator succeeds to reach the goal position in an environment cluttered with obstacles. The input trajectories imposed for the base are $\left[x^{*}(t), y^{*}(t), \vartheta^{*}(t)\right]^{T}=[0.1 t, 0.1 t, \pi / 4]^{T}$, the articular movement imposed for the arm is $q_{r}^{*}=\left[q_{r_{1}}^{*}, q_{r_{2}}^{*}\right]^{T}=$ $[\pi / 2,-\pi / 2]^{T}$. Figures $18-22$ illustrate the navigation obstacle avoidance strategy.

\section{Conclusion}

In this article we developed a new approach to control an omnidirectional mobile manipulator. Nonlinear equations of motion for the robot were derived including a kinematic model and a dynamic model. Based on these models, nonlinear control design for the robot was studied using the input-state linearization method. The robot model was linearized to obtain a linear model, and a linear controller was used to achieve tracking control of the robot position. The use of impedance control as an outer loop allows us to unify the control structure in the main case of robot interaction with the environment. Furthermore, the impedance adaptation allows us to overcome instability problems in terms of trajectory following.

The simulation results showed good behavior in the presence of obstacles for different values of the desired impedance. The perspectives of this work concern first the generalization of this process to add more functionalities such as physical cooperation with the humans. Impedance adaptation can give more flexibility in system control. Second, this work can be extended to the case of remote interaction with an operator. This interaction can give their the feeling of real experiment conditions, such as haptic feedbacks. All these tools must be integrated.
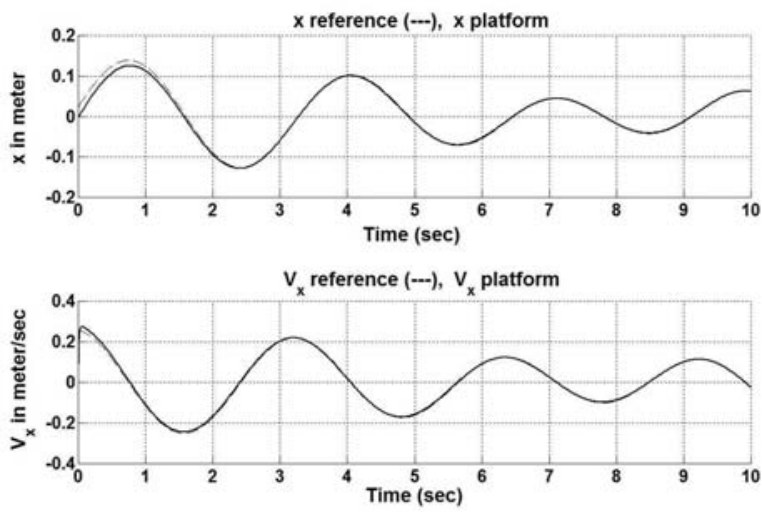

Fig. 13. Desired and measured $x$-trajectories, velocities of the platform.
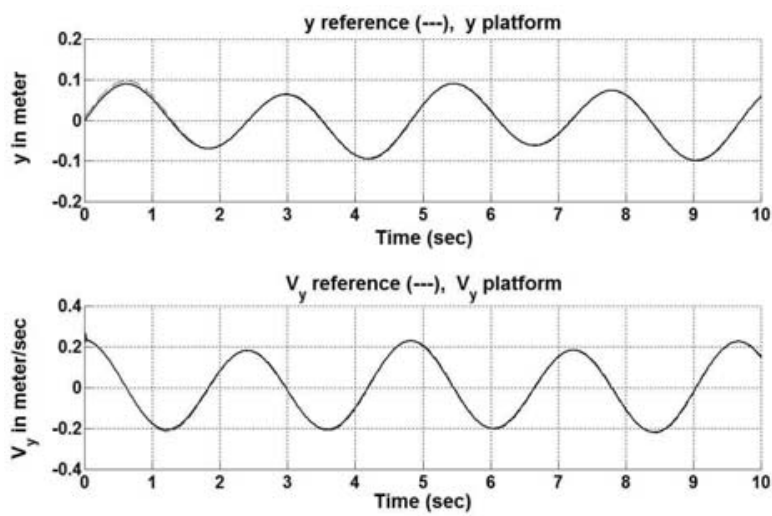

Fig. 14. Desired and measured $y$-trajectories, velocities of the platform.

\section{References}

Abdessemed, F. and Benmahammed, K. (2001). A two-layer robot controller design using evolutionnary algorithms, Journal of Intelligent and Robotic System 30(1): 73-94.

Abdessemed, F., Benmahammed, K. and Monacelli, E. (2004). A fuzzy-based reactive controller for a non-holonomic mobile robot, Journal of Robotics and Autonomous Systems 47(1): 31-46. 

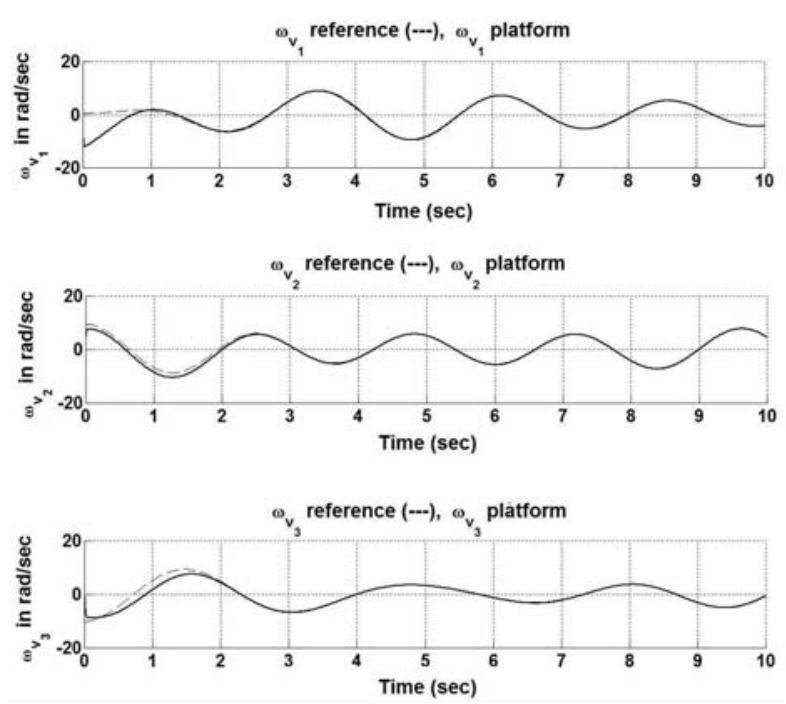

Fig. 15. Angular velocities $\omega_{v_{1}}, \omega_{v_{2}}$ and $\omega_{v_{3}}$.
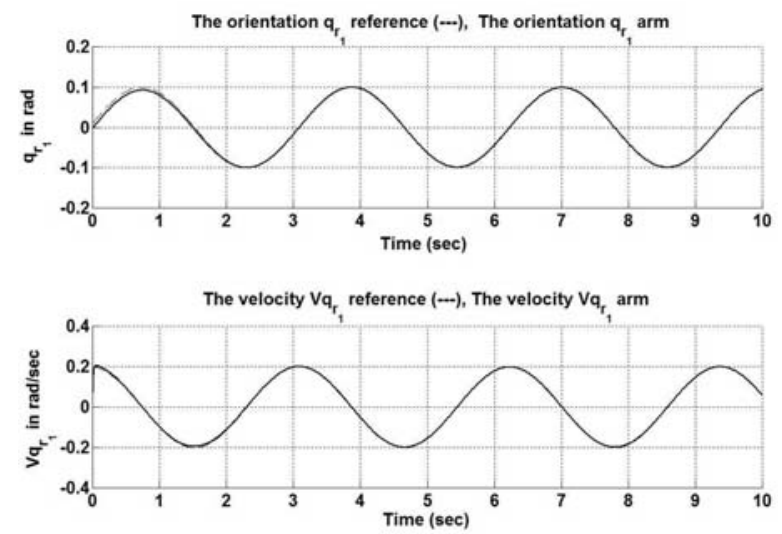

Fig. 16. Desired and measured $q_{r_{1}}$ angular trajectories, velocities of the arm.

Abdessemed, F., Benmahammed, K. and Monacelli, E. (2006). A learning paradigm for motion control of mobile manipulators, International Journal of Applied Mathematics and Computer Science 16(4): 475-484.

Albers, A., Brudniok, S., Ottnad, J., Sauter, C. and Sedchaicharn, K. (2006). Upper body of a new humanoid robot: The design of ARMAR 3, 6th IEEE-RAS International Conference on Humanoid Robots, Genova, Italy, pp. 308-313.

Ambrose, R.O., Savely, R.T., Goza, S.M., Strawser, P., Diftler, M.A., Spain, I., Radford, N. and Martin, L. (2004). Mobile manipulation using NASA's Robonaut, Proceedings of the IEEE International Conference on Robotics and Automation, ICRA'2004, New Orleans, LA, USA, Vol. 2, 21042109.

Arai, T. and Ota, J. (1996). Motion planning of multiple mobile robots using virtual impedance, Journal of Robotics and Mechatronics 8(1): 67-74.
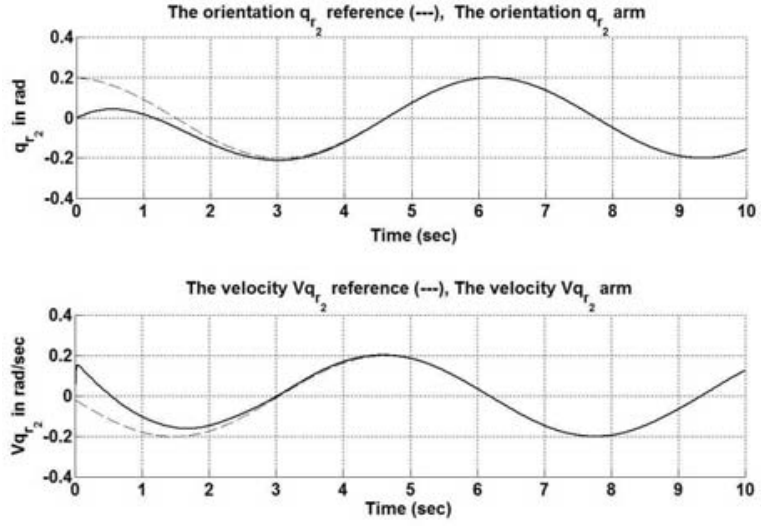

Fig. 17. Desired and measured $q_{r_{2}}$ angular trajectories, velocities of the arm.

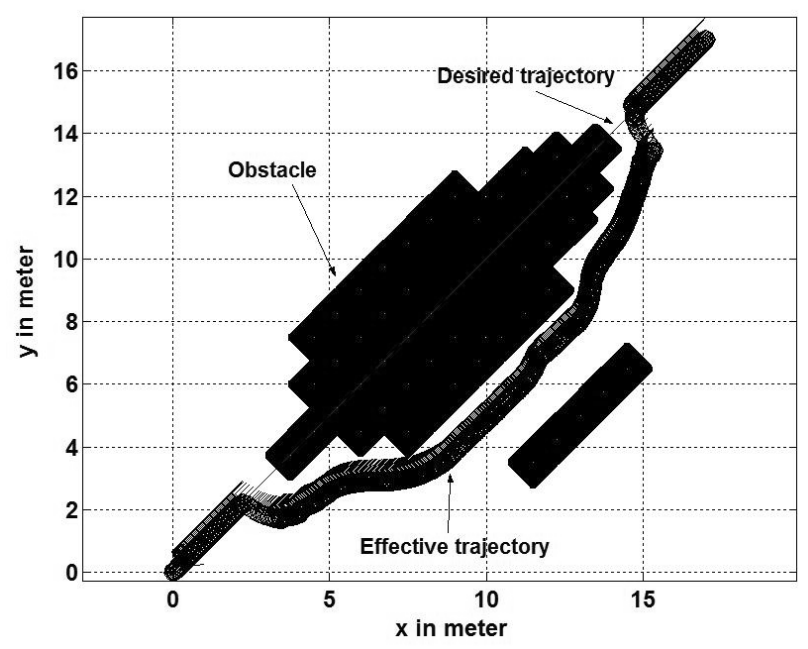

Fig. 18. Robot motion with a cluttered obstacle environment.

Bayle, B. (2001). Modélisation et commande cinématique des manipulateurs mobiles à roues, Ph.D. thesis, LAASCNRS, Toulouse University, Toulouse .

Bischoff, R. and Graefe, V. (2004). Hermes: A versatile personal assistant robot, Proceedings of the IEEE 92(11): 17591779 .

Borenstein, J. and Koren, Y. (1991). The vector field histogramfast obstacle avoidance for mobile robots, IEEE Journal of Robotics and Automation 7(3): 278-288.

Campion, G., Bastin, G. and D'Andréa-Novel, B. (1996). Structural proprieties and classification of kinematic and dynamic models of wheeled mobile robots, IEEE Transactions on Robotics and Automation 12(1): 47-62.

Carelli, R., Secchi, H. and Mut, V. (1999). Algorithms for stable control of mobile robots with obstacle avoidance, Latin American Applied Research 29(3/4): 191-196.

Djebrani, S. and Abdessemed, F. (2009). Multi-agent prototyping for a cooperative carrying task, IEEE International 

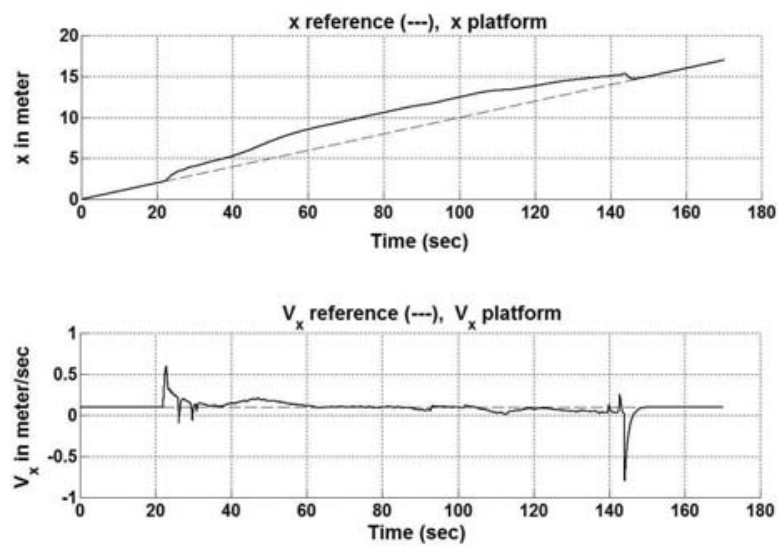

Fig. 19. Desired and measured $x$-trajectories, velocities of the platform.
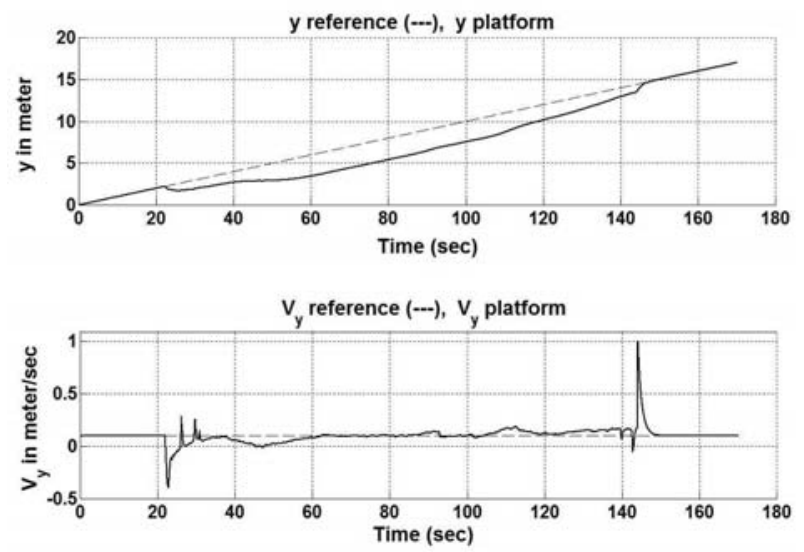

Fig. 20. Desired and measured $y$-trajectories, velocities of the platform.

Conference on Robotics and Biomimetics, ROBIO'2009, Guilin, Guangxi, China, pp. 1421-1426.

Djebrani, S., Benali, A. and Abdessemed, F. (2009). Forceposition control of a holonomic mobile manipulator, 12th International Conference on Climbing and Walking Robots and the Support Technologies for Mobile Machines, CLAWAR'2009, Istanbul, Turkey, pp. 1023-1030.

Djebrani, S., Abdessemed, F. and Benali, A. (2010a). A multiagent strategy for a simple cooperative behavior, International Journal of Information Acquisition 7(4): 331-345.

Djebrani, S., Benali, A. and Poisson, G. (2010b). Input-state linearisation of an omni-directional mobile robot, IEEE International Symposion on Industrial Electronics, ISIE'2010, Bary, Italy, pp. 1889-1894.

Djebrani, S., Benali, A. and Abdessemed, F. (2011). Modelling and feedback control of an omni-directional mobile manipulator, IEEE Conference on Automation Science and Engineering, CASE'2011, Trieste, Italy, pp. 785-791.
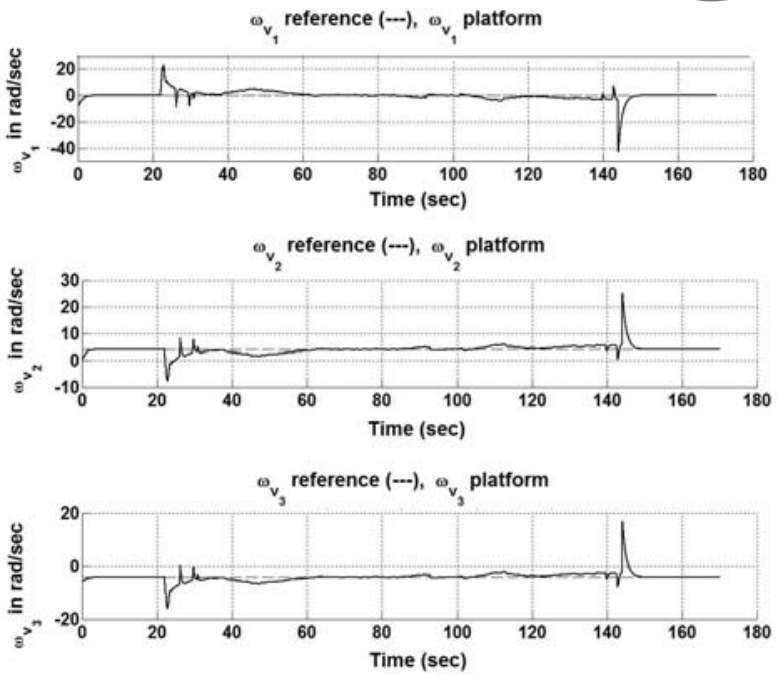

Fig. 21. Angular velocities $\omega_{v_{1}}, \omega_{v_{2}}$ and $\omega_{v_{3}}$.

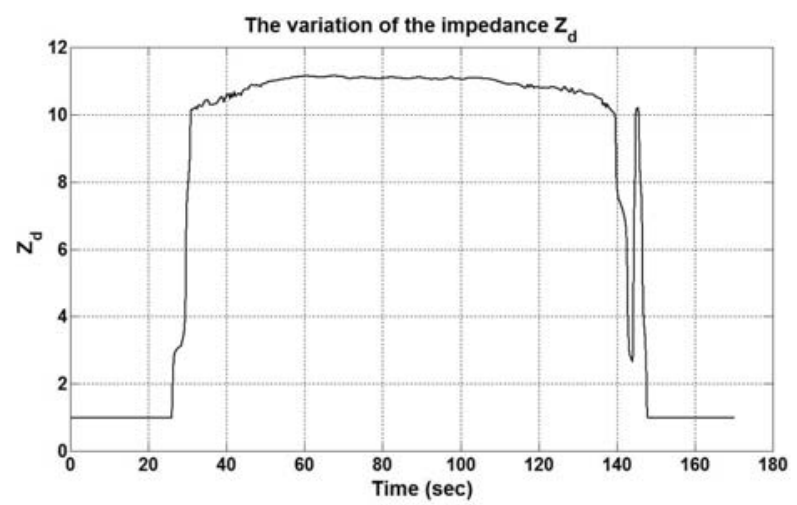

Fig. 22. Variation of impedance $Z_{d}$.

Goldenberg, A. A. (1988). Implementation of force and impedance control in robot manipulators, Proceedings of the 27th IEEE International Conference on Decision and Control, Philadelphia, PA, USA, Vol. 3, pp. 1626-1632.

Hashimoto, S. (2002). Humanoid robots in Waseda University: Hadalay-2 and Wabian, Journal of Autonomous Robots 12(1): 25-38.

Hogan, N. (1985). Impedance control: An approach to manipulation, I: Theory, II: Implementation, III: Applications, ASME Journal of Dynamic Systems, Measurement and Control 107(1): 1-24.

Isidori, A. (1995). Nonlinear Control Systems, 3rd Edn., Springer-Verlag, New York, NY.

Khalil, W. and Kleinfinger, J. (1986). A new geometric notation for open and closed loop robots, Proceedings of the IEEE International Conference on Robotics and Automation, ICRA'86, San Francisco, CA, USA, Vol. 3, pp. 1174 1180 . 
Khatib, O. (1986). Real-time obstacle avoidance for manipulators and mobile robots, International Journal of Robotics Research 5(1): 90-98.

Khatib, O., Yokoi, K., Chang, K., Ruspini, D., Holmberg, R. and Casal, A. (1996). Coordination and decentralized cooperation of multiple mobile manipulators, Journal of Robotic Systems 13(11): 755-764.

Konno, A., Nagashima, K., Furukawa, R., Nishiwaki, K., Noda, T., Inaba, M. and Inoue, H. (1997). Development of a humanoid robot Saika, Proceedings of the IEEE/RSJ International Conference on Intelligent Robots and Systems, IROS'97, Grenoble, France, pp. 805-810.

Kosuge, K., Sato, M. and Kazamura, N. (2000). Mobile robot helper, Proceedings of the IEEE International Conference on Robotics and Automation, ICRA'00, San Francisco, CA, USA, pp. 583-588.

Liu, K. and Lewis, F.L. (1990). Decentralized continuous robust controller for mobile robots, Proceedings of the IEEE International Conference on Robotics and Automation, ICRA'90, Cincinnati, OH, USA, Vol. 3, pp. 1822-1827.

Mourioux, G., Novales, C., Poisson, G. and Vieyres, P. (2006). Omni-directional robot with spherical orthogonal wheels: Concepts and analyses, Proceedings of the IEEE International Conference on Robotics and Automation, ICRA'06, Orlando, FL, USA, pp. 3374-3379.

Pin, F.G. and Killough, S.M. (1994). A new family of omnidirectional and holonomic wheeled platforms for mobile robots, IEEE Transactions on Robotics and Automation 10(4): 480-489.

Poisson, G., Parmantier, Y. and Novales, C. (2001). Modélisation cinématique d'unrobot mobile omnidirectionnel à roues sphériques, XVième Congrés Français de Mécanique, Nancy, France, pp. 3-7.

Sciavicco, L. and Siciliano, B. (2000). Modelling and Control of Robots Manipulators, 2nd Edn., Advanced Textbooks in Control and Signal Processing Series, Springer-Verlag, London.

Slotine, J. J. and Li, W. (1991). Applied Nonlinear Control, Prentice-Hall, Upper Saddle River, NJ

specAmotor (2011). Re-40-148867: Electric motor datasheet, http: / /www. specamotor.com/pdf/ datasheet_RE-40-148867_en.pdf.

Spong, M.W., Hutchinson, S. and Vidyasagar, M. (1989). Robot Dynamics and Control, John Wiley, New York, NY.

Sugeno, M. and Nishida, M. (1985). Fuzzy control of a model car, Fuzzy Sets and Systems 16(2): 103-113.

Watanabe, K., Shiraishi, Y., Tzafestas, S.G., Tang, J. and Fukuda, T. (1998). Feedback control of an omnidirectional autonomous platform for mobile service robots, Journal of Intelligent and Robotic Systems 22(3-4): 315-330.

Williams, R.L., Carter, B.E., Gallina, P. and Rosati, G. (2002). Dynamic model with slip for wheeled omnidirectional robots, IEEE Transactions on Robotics and Automation 18(3): 285-293.
Yamamoto, Y. (1994). Control and Coordination of Locomotion and Manipulation of a Wheeled Mobile Manipulator, $\mathrm{Ph} . \mathrm{D}$. thesis, University of Pennsylvania, Philadelphia, PA.

Salima Djebrani is currently a lecturer of electrical engineering at the University of Batna, Algeria. She has received a B.Sc. degree in electrical engineering (industrial control) and an M.Sc. degree, both from the University of Batna, in 1997 and 2002, respectively. She is currently a Ph.D. student in robotics. She has worked for two years at the schoo of ENSI de Bourges in France as part of a cotutelle Ph.D. Her research interests include robotics, multi-agent systems, intelligent systems and control.

Abderraouf Benali is currently an associate professor with the Depart ment of Electrical Engineering and the PRISME laboratory at Bourges, France. He received his diploma in electrical engineering from the Technical University of Algiers in 1988, and the M.Sc. and Ph.D. degrees in control and robotics from the University of Paris VI, France, in 1991 and 1997, respectively. From 1997 to 1999 he was a research assistant at the Robotic Laboratory of Paris. In 2000, he joined ENSI at Bourges, France. He is mainly interested in haptic interface control, sensor-based robot motion planning, and hybrid control systems.

Foudil Abdessemed is an associate professor at the University of Batna, Algeria. He had worked as an academic member for 2 years in the Robotic Laboratory of Paris in France, where he finished his Ph.D. His qualifications include a B.Sc., an M.Sc., and a Ph.D. in electrical engineering with honors. Through 16 years of teaching and research, he has gained in-depth knowledge of many scientific subjects and experience. He has been involved in many scientific and research projects and has directed some others. Work in the field of robotics has allowed him to develop skills in the areas of modern control, electronics, evolutionary algorithms, network, computer, communications, and robotics.

\section{Appendix}

The motion equation (27) gives

$$
\begin{aligned}
& {\left[\begin{array}{ll}
M_{r}^{(11)} & M_{r}^{(12)} \\
M_{r}^{(21)} & M_{r}^{(22)}
\end{array}\right]\left[\begin{array}{l}
\dot{\omega}_{r_{1}} \\
\dot{\omega}_{r_{2}}
\end{array}\right]} \\
& +\left[\begin{array}{ll}
C_{r_{1}}^{(11)} & C_{r_{1}}^{(12)} \\
C_{r_{1}}^{(21)} & C_{r_{1}}^{(22)}
\end{array}\right]\left[\begin{array}{l}
\omega_{r_{1}} \\
\omega_{r_{2}}
\end{array}\right]+\left[\begin{array}{c}
C_{r_{2}}^{(1)} \\
C_{r_{2}}^{(2)}
\end{array}\right] \\
& =\left[\begin{array}{l}
\tau_{r_{1}} \\
\tau_{r_{2}}
\end{array}\right]-\left[\begin{array}{lll}
R_{r}^{(11)} & R_{r}^{(12)} & R_{r}^{(13)} \\
R_{r}^{(21)} & R_{r}^{(22)} & R_{r}^{(23)}
\end{array}\right]\left[\begin{array}{l}
\dot{\omega}_{v_{1}} \\
\dot{\omega}_{v_{2}} \\
\dot{\omega}_{v_{3}}
\end{array}\right],
\end{aligned}
$$

where

$$
\begin{aligned}
& C_{r_{1}}^{(11)}=-m_{2} l_{1} l_{c_{2}} \sin \left(q_{r_{2}}\right) \omega_{r_{2}}, \\
& C_{r_{1}}^{(12)}=-m_{2} l_{1} l_{c_{2}} \sin \left(q_{r_{2}}\right)\left(\omega_{r_{1}}+\omega_{r_{2}}\right), \\
& C_{r_{1}}^{(21)}=+m_{2} l_{1} l_{c_{2}} \sin \left(q_{r_{2}}\right) \omega_{r_{1}}, \\
& C_{r_{1}}^{(22)}=0,
\end{aligned}
$$




$$
\begin{aligned}
C_{r_{2}}^{(1)}= & \left(-2 I_{2} l_{1} l_{2} \sin \left(q_{r_{2}}\right)\right) \omega_{r_{2}} \omega_{v_{3}}, \\
C_{r_{2}}^{(2)}= & \left(2 I_{2} l_{1} l_{2} \sin \left(q_{r_{2}}\right)\right) \omega_{r_{1}} \omega_{v_{3}}+\left(I_{2} l_{1} l_{2} \sin \left(q_{r_{2}}\right)\right) \omega_{v_{3}}^{2}, \\
R_{r}^{(11)}= & \left.-l_{2} \sin \left(q_{r_{1}}+q_{r_{2}}+\vartheta\right)-l_{1} \sin \left(q_{r_{1}}+\vartheta\right)\right] I_{2} \\
& -I_{1} l_{1} \sin \left(q_{r_{1}}+\vartheta\right), \\
R_{r}^{(12)}= & {\left[l_{2} \cos \left(q_{r_{1}}+q_{r_{2}}+\vartheta\right)+l_{1} \cos \left(q_{r_{1}}+\vartheta\right)\right] I_{2} } \\
& +I_{1} l_{1} \cos \left(q_{r_{1}}+\vartheta\right), \\
R_{r}^{(13)}= & {\left[\left(-l_{2} \sin \left(q_{r_{1}}+q_{r_{2}}+\vartheta\right)-l_{1} \sin \left(q_{r_{1}}+\vartheta\right)\right)^{2}\right.} \\
& +\left(l_{2} \cos \left(q_{r_{1}}+q_{r_{2}}+\vartheta\right)+l_{1} \cos \left(q_{r_{1}}+\vartheta\right)\right)^{2} \\
& +2] I_{2}+I_{1}\left(l_{1}^{2}+2\right), \\
R_{r}^{(21)}= & -I_{2} l_{2} \sin \left(q_{r_{1}}+q_{r_{2}}+\vartheta\right), \\
R_{r}^{(22)}= & +I_{2} l_{2} \cos \left(q_{r_{1}}+q_{r_{2}}+\vartheta\right), \\
R_{r}^{(23)}= & I_{2}\left[l_{1} l_{2} \cos \left(q_{r_{2}}\right)+l_{2}^{2}+2\right] .
\end{aligned}
$$

The motion equation (28) yield

$$
\begin{aligned}
& {\left[\begin{array}{lll}
M_{v_{1}}^{(11)} & M_{v_{1}}^{(12)} & M_{v_{1}}^{(13)} \\
M_{v_{1}}^{(21)} & M_{v_{1}}^{(22)} & M_{v_{1}}^{(23)} \\
M_{v_{1}}^{(31)} & M_{v_{1}}^{(32)} & M_{v_{1}}^{(33)}
\end{array}\right]\left[\begin{array}{l}
\dot{\omega}_{v_{1}} \\
\dot{\omega}_{v_{2}} \\
\dot{\omega}_{v_{3}}
\end{array}\right] } \\
+ & {\left[\begin{array}{lll}
C_{v_{1}}^{(11)} & C_{v_{1}}^{(12)} & C_{v_{1}}^{(13)} \\
C_{v_{1}}^{(21)} & C_{v_{1}}^{(22)} & C_{v_{1}}^{(23)} \\
C_{v_{1}}^{(31)} & C_{v_{1}}^{(32)} & C_{v_{1}}^{(33)}
\end{array}\right]\left[\begin{array}{l}
\omega_{v_{1}} \\
\omega_{v_{2}} \\
\omega_{v_{3}}
\end{array}\right]+\left[\begin{array}{l}
C_{v_{2}}^{(1)} \\
C_{v_{2}}^{(2)} \\
C_{v_{2}}^{(3)}
\end{array}\right] } \\
= & {\left[\begin{array}{lll}
E_{v}^{(11)} & E_{v}^{(12)} & E_{v}^{(13)} \\
E_{v}^{(21)} & E_{v}^{(22)} & E_{v}^{(23)} \\
E_{v}^{(31)} & E_{v}^{(32)} & E_{v}^{(33)}
\end{array}\right]\left[\begin{array}{l}
\tau_{v_{1}} \\
\tau_{v_{2}} \\
\tau_{v_{3}}
\end{array}\right] } \\
& -\left[\begin{array}{ccc}
M_{v_{2}}^{(11)} & M_{v_{2}}^{(12)} & M_{v_{2}}^{(13)} \\
M_{v_{2}}^{(21)} & M_{v_{2}}^{(22)} & M_{v_{2}}^{(23)} \\
M_{v_{2}}^{(31)} & M_{v_{2}}^{(32)} & M_{v_{2}}^{(33)}
\end{array}\right]\left[\begin{array}{l}
\dot{\omega}_{v_{1}} \\
\dot{\omega}_{v_{2}} \\
\dot{\omega}_{v_{3}}
\end{array}\right] \\
- & {\left[\begin{array}{lll}
R_{v}^{(11)} & R_{v}^{(12)} \\
R_{v}^{(21)} & R_{v}^{(22)} \\
R_{v}^{(31)} & R_{v}^{(32)}
\end{array}\right]\left[\begin{array}{l}
\dot{\omega}_{r_{1}} \\
\dot{\omega}_{r_{2}}
\end{array}\right], }
\end{aligned}
$$

where

$$
\begin{aligned}
M_{v_{1}}^{(11)}= & M_{v_{1}}^{(22)}=M_{v_{1}}^{(33)}=4 r I_{R}+\frac{4 m_{R} r}{9} \\
& +\frac{I_{m} n_{m}^{2}}{r} \\
M_{v_{1}}^{(12)}= & M_{v_{1}}^{(13)}=M_{v_{1}}^{(21)}=M_{v_{1}}^{(23)}=M_{v_{1}}^{(31)} \\
= & M_{v_{1}}^{(32)}=4 r I_{R}-\frac{2 m_{R} r}{9} \\
C_{v_{1}}^{(11)}= & C_{v_{1}}^{(22)}=C_{v_{1}}^{(33)}=\frac{C_{m} C_{e} n_{m}^{2}}{r R_{a}}+\frac{b_{m} n_{m}^{2}}{r}
\end{aligned}
$$

$$
\begin{aligned}
& C_{v_{1}}^{(12)}=C_{v_{1}}^{(23)}=C_{v_{1}}^{(31)}=\frac{-2 r m_{R} \dot{\vartheta}}{3 \sqrt{3}}, \\
& C_{v_{1}}^{(13)}=C_{v_{1}}^{(21)}=C_{v_{1}}^{(32)}=\frac{2 r m_{R} \dot{\vartheta}}{3 \sqrt{3}}, \\
& C_{v_{2}}^{(1)}=-2 I_{1} l_{1} \cos \left(q_{r_{1}}+\vartheta\right) \omega_{r_{1}} \omega_{v_{3}}, \\
& -2 I_{2}\left[l_{2} \cos \left(q_{r_{1}}+q_{r_{2}}+\vartheta\right)\right. \\
& \left.+l_{1} \cos \left(q_{r_{1}}+\vartheta\right)\right] \omega_{r_{1}} \omega_{v_{3}} \\
& -2 I_{2} l_{2} \cos \left(q_{r_{1}}+q_{r_{2}}+\vartheta\right) \omega_{r_{2}} \omega_{v_{3}} \\
& -I_{1} l_{1} \cos \left(q_{r_{1}}+\vartheta\right) \omega_{r_{1}}^{2} \\
& -I_{2}\left[l_{2} \cos \left(q_{r_{1}}+q_{r_{2}}+\vartheta\right)\right. \\
& \left.+l_{1} \cos \left(q_{r_{1}}+\vartheta\right)\right] \\
& \omega_{r_{1}}^{2}-I_{2} l_{2} \cos \left(q_{r_{1}}+q_{r_{2}}+\vartheta\right) \\
& \omega_{r_{1}} \omega_{r_{2}}-I_{2} l_{2} \cos \left(q_{r_{1}}+q_{r_{2}}+\vartheta\right) \\
& \omega_{r_{2}} \omega_{r_{1}}-I_{2} l_{2} \cos \left(q_{r_{1}}+q_{r_{2}}+\vartheta\right) \omega_{r_{2}}^{2}, \\
& C_{v_{2}}^{(2)}=-2 I_{1} l_{1} \sin \left(q_{r_{1}}+\vartheta\right) \omega_{r_{1}} \omega_{v_{3}} \\
& -2 I_{2}\left[l_{2} \sin \left(q_{r_{1}}+q_{r_{2}}+\vartheta\right)\right. \\
& \left.+l_{1} \sin \left(q_{r_{1}}+\vartheta\right)\right] \omega_{r_{1}} \omega_{v_{3}} \\
& -2 I_{2} l_{2} \sin \left(q_{r_{1}}+q_{r_{2}}+\vartheta\right) \omega_{r_{2}} \omega_{v_{3}} \\
& -I_{1} l_{1} \sin \left(q_{r_{1}}+\vartheta\right) \omega_{r_{1}}^{2} \\
& -I_{2}\left[l_{2} \sin \left(q_{r_{1}}+q_{r_{2}}+\vartheta\right)\right. \\
& \left.+l_{1} \sin \left(q_{r_{1}}+\vartheta\right)\right] \omega_{r_{1}}^{2} \\
& -I_{2} l_{2} \sin \left(q_{r_{1}}+q_{r_{2}}+\vartheta\right) \omega_{r_{1}} \omega_{r_{2}} \\
& -I_{2} l_{2} \sin \left(q_{r_{1}}+q_{r_{2}}+\vartheta\right) \omega_{r_{2}} \omega_{r_{1}} \\
& -I_{2} l_{2} \sin \left(q_{r_{1}}+q_{r_{2}}+\vartheta\right) \omega_{r_{2}}^{2}, \\
& C_{v_{2}}^{(3)}=-2 I_{2} l_{1} l_{2} \sin \left(q_{r_{2}}\right) \omega_{r_{2}} \omega_{v_{3}} \\
& -I_{2} l_{1} l_{2} \sin \left(q_{r_{2}}\right) \omega_{r_{1}} \omega_{r_{2}} \\
& -I_{2} l_{1} l_{2} \sin \left(q_{r_{2}}\right) \omega_{r_{2}} \omega_{r_{1}} \\
& -I_{2} l_{1} l_{2} \sin \left(q_{r_{2}}\right) \omega_{r_{2}} \text {, } \\
& E_{v}^{(11)}=E_{v}^{(22)}=E_{v}^{(33)}=\frac{C_{m} n_{m}}{r R_{a}}, \\
& E_{v}^{(12)}=E_{v}^{(13)}=E_{v}^{(21)}=E_{v}^{(23)}=E_{v}^{(31)} \\
& =E_{v}^{(32)}=0, \\
& M_{v_{2}}^{(11)}=I_{1}+I_{2} \text {, } \\
& M_{v_{2}}^{(12)}=0 \text {, } \\
& M_{v_{2}}^{(13)}=\left[-l_{2} \sin \left(q_{r_{1}}+q_{r_{2}}+\vartheta\right)-l_{1} \sin \left(q_{r_{1}}+\vartheta\right)\right] I_{2} \\
& -I_{1} l_{1} \sin \left(q_{r_{1}}+\vartheta\right), \\
& M_{v_{2}}^{(21)}=0 \text {, } \\
& M_{v_{2}}^{(22)}=I_{1}+I_{2} \text {, } \\
& M_{v_{2}}^{(23)}=\left[l_{2} \cos \left(q_{r_{1}}+q_{r_{2}}+\vartheta\right)+l_{1} \cos \left(q_{r_{1}}+\vartheta\right)\right] I_{2} \\
& +I_{1} l_{1} \cos \left(q_{r_{1}}+\vartheta\right) \text {, } \\
& M_{v_{2}}^{(31)}=\left[-l_{2} \sin \left(q_{r_{1}}+q_{r_{2}}+\vartheta\right)-l_{1} \sin \left(q_{r_{1}}+\vartheta\right)\right] I_{2} \\
& -I_{1} l_{1} \sin \left(q_{r_{1}}+\vartheta\right) \text {, }
\end{aligned}
$$




$$
\begin{array}{rlrl}
M_{v_{2}}^{(32)}= & {\left[+l_{2} \cos \left(q_{r_{1}}+q_{r_{2}}+\vartheta\right)+l_{1} \cos \left(q_{r_{1}}+\vartheta\right)\right] I_{2}} & R_{v}^{(21)}= & {\left[+l_{2} \cos \left(q_{r_{1}}+q_{r_{2}}+\vartheta\right)+l_{1} \cos \left(q_{r_{1}}+\vartheta\right)\right] I_{2}} \\
& +I_{1} l_{1} \cos \left(q_{r_{1}}+\vartheta\right), & & +I_{1} l_{1} \cos \left(q_{r_{1}}+\vartheta\right), \\
M_{v_{2}}^{(33)}= & {\left[\left(-l_{2} \sin \left(q_{r_{1}}+q_{r_{2}}+\vartheta\right)-l_{1} \sin \left(q_{r_{1}}+\vartheta\right)\right)^{2}\right.} & R_{v}^{(22)}= & +I_{2} l_{2} \cos \left(q_{r_{1}}+q_{r_{2}}+\vartheta\right), \\
& +\left(l_{2} \cos \left(q_{r_{1}}+q_{r_{2}}+\vartheta\right)+l_{1} \cos \left(q_{r_{1}}+\vartheta\right)\right)^{2} & R_{v}^{(31)}=\left[\left(-l_{2} \sin \left(q_{r_{1}}+q_{r_{2}}+\vartheta\right)-l_{1} \sin \left(q_{r_{1}}+\vartheta\right)\right)^{2}\right. \\
& +2] I_{2}+I_{1}\left(l_{1}^{2}+2\right), & & +\left(l_{2} \cos \left(q_{r_{1}}+q_{r_{2}}+\vartheta\right)+l_{1} \cos \left(q_{r_{1}}+\vartheta\right)\right)^{2} \\
R_{v}^{(11)}= & {\left[-l_{2} \sin \left(q_{r_{1}}+q_{r_{2}}+\vartheta\right)-l_{1} \sin \left(q_{r_{1}}+\vartheta\right)\right] I_{2}} & & +2] I_{2}+I_{1}\left(l_{1}^{2}+2\right), \\
& -I_{1} l_{1} \sin \left(q_{r_{1}}+\vartheta\right), & R_{v}^{(32)}= & I_{2}\left[l_{1} l_{2} \cos \left(q_{r_{2}}\right)+l_{2}^{2}+2\right] . \\
R_{v}^{(12)}= & -I_{2} l_{2} \sin \left(q_{r_{1}}+q_{r_{2}}+\vartheta\right), & &
\end{array}
$$

